\title{
35. DATA REPORT: INORGANIC MAJOR, TRACE, AND RARE EARTH ELEMENT ANALYSES OF THE MUDS AND MUDSTONES FROM SITE $808^{1}$
}

\author{
Kevin T. Pickering, ${ }^{2}$ Nicholas G. Marsh, ${ }^{2}$ and Brian Dickie ${ }^{2}$
}

\section{OBJECTIVES}

The principal aims of undertaking a shore-based bulk inorganic geochemical analysis of muds and mudstones from Site 808 were as follows:

1. Characterize the geochemical signature of the muds and mudstones at regular intervals downhole to sample and identify any changes in sediment type and provenance.

2. Integrate the inorganic geochemistry with the shipboard and more detailed land-based laboratory studies of the clay minerals.

3 . Investigate any possible inorganic geochemical anomalies associated with the décollement.

\section{ANALYTICAL TECHNIQUES}

Major- and trace-element analysis of the samples was carried out at the University of Leicester Department of Geology by X-ray fluorescence (XRF) spectrometry using an ARL8420+ XRF spectrometer and a Philips PW1400 XRF spectrometer. Inductively coupled plasma/optical emission (ICP/OE) spectrometry was used to determine a more complete range of rare earth elements (REE). These analyses were carried out on the University of Leicester Department of Geology Philips PV8060 simultaneous ICP/OE spectrometer. Due to the small amounts of material available for many of the samples, the techniques developed and routinely used at Leicester for geochemical analysis of igneous samples from DSDP and ODP Legs had to be modified (see, for example, Marsh et al., 1980, Marsh et al., 1983, Saunders et al., 1991, Tarney and Marsh, 1991). Hence, a more detailed description of the methodology is presented.

\section{SAMPLE PREPARATION}

All samples were checked against the original sample lists and then given an abbreviated laboratory number that also introduced a degree of randomization to the sample processing order. All samples were then dried overnight at $105^{\circ} \mathrm{C}$ after removal from their plastic sampling tubes where necessary. The samples were then crushed to an average particle size of between 1 and $2 \mathrm{~mm}$ by crushing between the hardened steel faces of a "fly-press." Previous work with igneous and metamorphic rocks has shown contamination to be minimal by this technique. Due to the small amounts of material available, the routine crushing technique of using an agate swing mill was not viable. Hence the "fly-pressed" samples were crushed for 35-40 min in a Fritsch Pulverisette agate vibratory ball pestle and mortar to reduce the samples to fine powders (average grain size $<200 \mu \mathrm{m}$ mesh). The resultant fine powders were then dried again overnight at $105^{\circ} \mathrm{C}$. Prior to use for XRF sample preparation or ICP/OES REE analysis sample digestion, the samples were ignited in platinum cru-

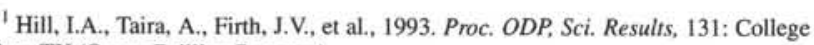
Station, TX (Ocean Drilling Program).

${ }^{2}$ Department of Geology, University of Leicester, Leicester LE1 7RH, United Kingdom.
}

cibles in a vented muffle furnace at $950^{\circ} \mathrm{C}$ for $90 \mathrm{~min}$. Losses on ignition were determined and are included in the data tables. The data for other elements have been recalculated to include the loss on ignition values.

\section{XRF ANALYSIS}

Major elements were determined on fusion disks prepared as follows. $1 \mathrm{~g}$ of ignited (volatile-free, fully oxidized) sample was fused with $5 \mathrm{~g}$ of Englehard Standard XRF grade Flux, an 80:20 mix of lithium metaborate and lithium tetraborate (equivalent to Johnson Matthey Spectroflux JM100B) for $20 \mathrm{~min}$ at $1100^{\circ} \mathrm{C}$ with intermittent swirling to ensure thorough mixing. The resultant melt was cast and pressed between the polished aluminum faces of a press, similar to the technique described by Harvey et al. (1973). The resultant fusion disks were then annealed at $250^{\circ} \mathrm{C}$ for a minimum of $3 \mathrm{hr}$. Analysis was performed on the University of Leicester Department of Geology ARL8420+ XRF spectrometer running a 3-kW rhodium anode X-ray tube at $50 \mathrm{kV} 50 \mathrm{~mA}$. Full analytical details are given in Table 1. Note that with the improved resolution of this spectrometer's design, it is possible to analyze for $\mathrm{P}$ using the $\mathrm{P} \mathrm{K} \alpha$ peak and pentaerythritol 002 (PET) as the analyzing crystal with no detectable overlap from the second order $\mathrm{Ca} \mathrm{K} \beta$ peak or its escape peak without having to resort to using $\mathrm{Ge}_{111}$.

The count data were collected on a dedicated IBM Series 2/70 computer and processed using ARL386 Phase 1 software. Calibrations have previously been produced from data collected on some 65 international reference materials and computing Traille-Lachancebased correction coefficients using the ARL386 software regression package. Values for the concentrations of the reference materials were taken from Govindaraju (1989).

Table 2 lists results for selected reference materials run at the same time as the samples to allow the estimation of precision and accuracy of the results. Our laboratory generally applies a quality control filter on major element analyses, only taking analyses with totals between $99.00 \%$ and $100.50 \%$ as acceptable. If analyses fall outside this range repeat fusion disks are prepared and analyzed. If the repeat analyses are within the acceptance range they are then used, or if triplicate beads reproduce analysis results within the expected precision range for each component, then the mean analysis for the triplicates is accepted in the event of totals falling below the acceptable range. Unfortunately the lack of sample in most cases precluded our usual quality checking, and in virtually all cases, the first-attempt analysis has had to stand. Certain samples did not have sufficient material to provide $1 \mathrm{~g}$ of ignited material for the fusion disk preparation described above. In these cases $0.5 \mathrm{~g}$ of ignited powder and $0.5 \mathrm{~g}$ of Koch Chemicals $99.999 \% \mathrm{SiO}_{2}$ were used to make fusion disks. The resulting analysis then had the error of the total from 100\% split 50:50 between $50.00 \%$ of the $\mathrm{SiO}_{2}$ content and the remainder of the component concentrations. The error-corrected $50 \% \mathrm{SiO}_{2}$ was then subtracted from the total $\mathrm{SiO}_{2}$ content and the remaining $\mathrm{SiO}_{2}$ content and other component concentrations were then doubled to generate the "sample" analysis. Blanks with just the Koch Chemicals 99.999\% $\mathrm{SiO}_{2}$ were also made to ensure that no detectable contamination from other elements was being introduced. 
Table 1. Analytical conditions for major-element analysis of fusion disks on the University of Leicester Department of Geology ARL8420+ XRF spectrometer.

\begin{tabular}{|c|c|c|c|c|c|c|c|c|c|c|}
\hline Element & Line & Crystal & Peak angle & $\begin{array}{l}\text { Count time } \\
\text { (s) }\end{array}$ & $\begin{array}{c}\text { Background } 1 \\
\text { angle }\end{array}$ & $\begin{array}{c}\text { Count time } \\
\text { (s) }\end{array}$ & $\begin{array}{c}\text { Background } 2 \\
\text { angle }\end{array}$ & $\begin{array}{l}\text { Count time } \\
\text { (s) }\end{array}$ & Collimator & Detector \\
\hline \multicolumn{11}{|l|}{ Goniometer 1} \\
\hline $\mathrm{P}$ & $\mathrm{Kal}, 2$ & PET & 89.54 & 50 & 91.97 & 25 & 86.7 & 25 & Fine & FPC \\
\hline $\mathrm{Si}$ & $\mathrm{Kal}, 2$ & PET & 109.21 & 20 & 112.44 & 5 & 105.57 & 5 & Std. Coarse & FPC \\
\hline $\mathrm{Al}$ & $\mathrm{Kal}, 2$ & PET & 145.12 & 30 & 148.85 & 15 & 140.44 & 15 & Std. Coarse & FPC \\
\hline $\mathrm{Mg}$ & $\mathrm{Kal}, 2$ & AX06 & 20.2 & 50 & 22.25 & 20 & 18.98 & 20 & Std. Coarse & FPC \\
\hline $\mathrm{Na}$ & $\mathrm{Kal}, 2$ & AX06 & 24.37 & 50 & 26.99 & 20 & 22.25 & 20 & Std. Coarse & FPC \\
\hline \multicolumn{11}{|l|}{ Goniometer 2} \\
\hline $\mathrm{Fe}$ & $\mathrm{Kal}, 2$ & LiF200 & 57.52 & 25 & 60.42 & 10 & 55.25 & 10 & Fine & FPC \\
\hline $\mathrm{Ti}$ & $\mathrm{Kal}, 2$ & LiF200 & 86.14 & 40 & 88.8 & 20 & 84.25 & 20 & Fine & FPC \\
\hline $\mathrm{Ca}$ & $\mathrm{Kal}, 2$ & LiF200 & 113.09 & 25 & 115.1 & 10 & 110.1 & 10 & Fine & FPC \\
\hline $\mathrm{K}$ & $\mathrm{Kal}, 2$ & LiF200 & 136.69 & 50 & 139.36 & 20 & 131.67 & 20 & Fine & FPC \\
\hline $\mathrm{Mn}$ & $\mathrm{Kal}, 2$ & $\mathrm{LiF} 220$ & 95.2 & 50 & 98 & 25 & 92.85 & 25 & Fine & FPC \\
\hline
\end{tabular}

Trace elements are routinely determined on pressed powder briquettes in our laboratory. Yet again the small amounts of material available precluded this approach. As a result, trace-element determinations were made on the fusion disks. $\mathrm{Nb}, \mathrm{Zr}, \mathrm{Y}, \mathrm{Sr}, \mathrm{Rb}, \mathrm{Ga}, \mathrm{Zn}, \mathrm{Ni}$, and Th were analyzed on the Philips PW1400 using a 3-kW Cr anode X-ray tube operated at $80 \mathrm{kV} 30 \mathrm{~mA}$. Sc, V, Cr, Co, Cu, Ba, La, Ce, and Nd were analyzed on the ARL8420+ using a $3 \mathrm{~kW}$ Rh anode $\mathrm{X}$-ray tube operated at $60 \mathrm{kV} 45 \mathrm{~mA}$. The following overlaps were corrected for $\mathrm{Sr} \mathrm{K} \beta$ on $\mathrm{Zr} \mathrm{K} \alpha, \mathrm{Rb} \mathrm{K} \beta$ on $\mathrm{Y} \mathrm{K} \alpha$, Ti $\mathrm{K} \beta$ on $\mathrm{V} \mathrm{K} \alpha$, followed by corrected $\mathrm{V} \mathrm{K} \beta$ on $\mathrm{Cr} \mathrm{K} \alpha$, Fe $\mathrm{K} \beta$ on $\mathrm{Co} \mathrm{K} \alpha$, and the mutual overlap of $\mathrm{Ce} \mathrm{Lb}_{1}$ and $\mathrm{Nd} \mathrm{La}$ on each other. Although $\mathrm{Ba}$ is determined on the $\mathrm{La}_{1}$ line, use of $\mathrm{LiF}_{220}$ on the ARL8420+ gives a line free from interference from both Ti $K \alpha$ and $\mathrm{Sc} K \beta$ at the concentration levels found in most geological samples. Similarly, the Sc $\mathrm{K} \alpha$ peak is free from overlap of $\mathrm{Ca} \mathrm{K} \beta$ at the concentrations of $\mathrm{Ca}$ found in most geological materials except limestones, some high $\mathrm{Ca}$ dolostones, and high $\mathrm{Ca}$ marbles.

Details of analytical conditions for each element are given in Table 3. Mass absorption correction was performed using mass attenuation coefficients calculated at each analyte wavelength from the major-element analyses and the values tabulated by Thinh and Leroux (1979). Calibrations were prepared from the fully corrected count data collected on 51 International Reference materials using the values given by Govindaraju (1989) except that for values of $\mathrm{Zr}$ and $\mathrm{Nb}$ the values given by Jochum et al. (1990) were used in preference. Table 4 gives details of precision and allows a comparison of accuracy for trace element determinations performed on a selection of reference materials analyzed with the samples. Table 5 gives the XRF analyses for the Site 808 samples.

While most of the samples analyzed fell well within the range of materials and concentration ranges covered by our selection of International Reference materials, this is not the case for Samples 131808C-83R-2, 57-58 cm, 131-808C-85R-1, 59-61 cm, and 131-808C$86 \mathrm{R}-2,110-112 \mathrm{~cm}$. Accordingly, the results quoted for these samples should be treated with a greater degree of caution.

\section{ICP ANALYSIS}

Simultaneous analysis of the REE was carried out using the University of Leicester Department of Geology Philips PV8060 ICP optical emission spectrometer. The technique is essentially the same as that described by Walsh et al. (1981); $0.5 \mathrm{~g}$ of ignited powder for each of the selected samples was digested in open PTFE beakers by $15 \mathrm{~mL} \mathrm{HF}$ plus $4 \mathrm{~mL} \mathrm{HClO}_{4}$, followed by an additional $4 \mathrm{~mL}$ aliquot of $\mathrm{HClO}_{4}$ on reaching incipient dryness, to ensure complete removal of excess $\mathrm{HF}$ and $\mathrm{Si}$ complexes. On drying to incipient dryness again, the samples are taken up in $20 \mathrm{~mL}$ of $25 \% \mathrm{HCl}$ and then diluted to $50 \mathrm{~mL}$ with reverse osmosis/deionized $\mathrm{H}_{2} \mathrm{O}$ prior to the REE's being separated by ion exchange chromatography in batches of 10 , each batch accompanied by two out of three reference materials (JB-1a,
SO-1, and SO-2) and a reagent blank. The columns used for the reference sample and reagent blank were selected at random for each batch. All the samples from each batch including the reagent blanks and the reference materials were analyzed in one group on the ICP spectrometer. The reagent blanks in each case showed no detectable contamination for REE's in all cases. Results for the reference materials are listed in Table 6, and the analyses are given in Table 7.

\section{RESULTS AND CONCLUSIONS}

Figure 1 presents the major and trace elements as abundance normalized to $\mathrm{Al}_{2} \mathrm{O}_{3}$ vs. depth, because all the analyzed samples were muds or mudrocks. The late stage at which the analyses presented in this report became available for integration into the other Leg 131 geochemical and mineralogical data sets, both from the aqueous phase geochemistry and clay mineralogical studies, preclude a rigorous interpretation of the significance of the data. Some preliminary interpretations are made elsewhere in this volume (Underwood et al., a,b, this volume).

In summary, the initial principal conclusions are as follows:

1. Throughout Site 808 , from the deepest Shikoku Basin hemipelagites to the youngest trench and slope muds, the bulk geochemical signature remains essentially uniform, apart from the interval at about $1060-1110 \mathrm{~m}$ below seafloor (mbsf) (Fig. 2). The inference from this is that the main sediment source for the muds remained constant over the past 14 m.y., i.e., from the Izu Collision Zone and the mainland Japan or Honshu arc.

2. The décollement is not marked by a significant change in the major- or trace-element inorganic geochemistry of the muds.

3. The décollement is associated with an anomaly in the heavy REE relative to the lighter REE (the subject of further current research). The enhanced heavy REE are associated with otherwise typical muds. A potential candidate for supplying the enriched REE abundances associated with the décollement could be the umber-like metalliferous sediments between about 1060 and 1110 mbsf (see below), i.e., their subcreted equivalents. Subcretion of this material, associated with the complete smectite-illite transformation to release low-chloride fluids and the changing Eh-pH/P-T conditions, may have combined to mobilize the rare earth elements from the umbers. As these postulated fluids moved along the overpressured, dynamically sealed, décollement toward the toe of the accretionary prism, the changing physicochemical conditions may well have induced substantially decreased solubilities for the heavy REE in particular, and therefore led to their preferential adsorption within the hemipelagic muds in the décollement.

4. At about $1060-1110$ mbsf, there is an interval of brown muds enriched in $\mathrm{Ca}, \mathrm{Fe}, \mathrm{Mg}, \mathrm{Mn}$, and Poxides, plus several trace elements, particularly Y, Sr, Th, Sc, Cu, Ba, and the REE(Figs. 1-3). We interpret 
this interval as an umber, probably created by hydrothermal circulation above the Shikoku Basin spreading center at about 12-10 Ma during the last phase of north-south extension in the Shikoku Basin (Chamot-Rooke et al., 1987). These sediments appear similar to ophiolitic umbers (cf. Robertson and Hudson, 1974) with concentrations of carbonate minerals. At ocean ridge spreading centers, ferromanganoan oxyhydroxides, occurring as deep-sea nodules or crusts, readily accommodate the REE (Fleet, 1984).

\section{ACKNOWLEDGMENTS}

The authors wish to thank Addy Holmes, Rob Kelly, and Vincent Hilton for all their assistance with sample preparation and aid in preparing this data set. The authors also wish to acknowledge the financial support of the Department of Geology at the University of Leicester, which enabled them to perform these analyses.

\section{REFERENCES*}

Chamot-Rooke, N., Renard, V., and Le Pichon, X., 1987. Magnetic anomalies in the Shikoku Basin: a new interpretation. Earth Planet. Sci. Lett., $83: 214-228$.

Fleet, A.J., 1984. Aqueous and sedimentary geochemistry of the rare earth elements. In Henderson, P. (Ed.), Rare Earth Element Geochemistry: Amsterdam (Elsevier), 343-373.

Govindaraju, K., 1989. Spec. Iss. Geostds. Newsl., 13.

Harvey, P.K., Taylor, D.M., Hendry, R.D., and Bancroft, F., 1973. An accurate fusion method for the analysis of rocks and chemically related materials by X-ray Fluorescence Spectrometry. X-ray Spectrom., 2:33-43.

Jochum, K.P., Seufert, H.M., and Thirlwall, M.F., 1990. High-sensitivity Nb analysis by spark-source mass spectrometry (SSMS) and calibration of XRF Nb and Zr. Chem. Geol., 81:1-16.

Marsh, N.G., Saunders, A.D., Tarney, J., and Dick, H.J.B., 1980. Geochemistry of basalts from the Shikoku and Daito Basins. In Klein, G.deV., Kobayashi, K., et al., Init. Repts. DSDP, 58: Washington (U.S. Govt. Printing Office), $805-842$.
Marsh, N.G., Tarney, J., and Hendry, G.L., 1983. Trace element geochemistry of basalts from Hole 504B, Panama Basin, Deep Sea Drilling Project Legs 69 and 70. In Cann, J.R., Langseth, M.G., Honnorez, J., Von Herzen, R.P., White, S.M., et al., Init. Repts. DSDP, 69: Washington (U.S. Govt. Printing Office), 747-763.

Nakamura, N., 1974. Determination of REE, Ba, Fe, Mg, Na and $\mathrm{K}$ in carbonaceous and ordinary chondrites. Geochim. Cosmochim. Acta, 44:1917-1930.

Robertson, A.H.F., and Hudson, J.D., 1974. Pelagic sediments in the Cretaceous and Tertiary history of the Troodos Massif, Cyprus. In Hsü, K.J., and Jenkyns, H.C. (Eds.), Pelagic Sediments on Land and Sea. Spec. Publ. Int. Assoc. Sedimentol., 403-436.

Saunders, A.D., Storey, M., Gibson, I.L., Leat, P., Hergt, J., and Thompson, R.N., 1991. Chemical and isotopic constraints on the origin of basalts from Ninetyeast Ridge, Indian Ocean: results from DSDP Legs 22 and 26 and ODP Leg 121. In Weissel, J., Peirce, J., Taylor, E., Alt, J., et al., Proc. ODP, Sci. Results, 121: College Station, TX (Ocean Drilling Program).

Tarney, J., and Marsh, N.G., 1991. Major and trace element geochemistry of Holes CY-1 and CY-4: implications for petrogenetic models. In Gibson, I.L., Malpas, J., Robinson, P.T., and Xenophontos, C. (Eds.), Init. Repts. Cyprus Crustal Study Project. Pap.-Geol. Surv. Can., 90-20:133-176.

Thinh, T.P., and Leroux, J., 1979. New basic empirical expression for computing tables of X-ray Mass Attenuation Coefficients. X-ray Spectrom., 8:85-91.

Walsh, J.N., Buckley, F., and Barker, J., 1981. The simultaneous determination of the rare earth elements in rocks using inductively coupled plasma source spectrometry. Chem. Geol., 33:141-153.

\footnotetext{
* Abbreviations for names of organizations and publication titles in ODP reference lists follow the style given in Chemical Abstracts Service Source Index (published by American Chemical Society).
}

Date of initial receipt: 23 June 1992

Date of acceptance: 3 September 1992

Ms 131SR-144 
Table 2. Mean values and 1-sigma standard deviations for the major elements determined on the ARL8420+ from a selection of reference materials analyzed with the Site 808 samples.

\begin{tabular}{|c|c|c|c|c|c|c|c|c|c|c|c|c|}
\hline \multirow[b]{2}{*}{ Reference } & \multicolumn{4}{|c|}{ Sample } & \multicolumn{4}{|c|}{ BOB-1 } & \multicolumn{4}{|c|}{ NIM-G } \\
\hline & Mean & Std. dev. & \%Std. dev. & $\begin{array}{l}\text { Number } \\
\text { of runs }\end{array}$ & Mean & Std. dev. & \%Std. dev. & $\begin{array}{l}\text { Number } \\
\text { of runs }\end{array}$ & Mean & Std. dev. & \%Std. dev. & $\begin{array}{l}\text { Number } \\
\text { of runs }\end{array}$ \\
\hline $\mathrm{SiO}_{2}$ & 50.59 & 0.1 & 0.2 & 10 & 77.75 & 0.49 & 0.63 & 5 & 66.06 & 0.27 & 0.41 & 5 \\
\hline $\mathrm{TiO}_{2}$ & 1.29 & 0.05 & 0.35 & 10 & 0.1 & 0 & 0.2 & 5 & 0.88 & 0 & 0.57 & 5 \\
\hline $\mathrm{Al}_{2} \mathrm{O}_{3}$ & 16.4 & 0.04 & 0.24 & 10 & 11.52 & 0.07 & 0.63 & 5 & 14.35 & 0.05 & 0.32 & 5 \\
\hline $\mathrm{Fe}_{2} \mathrm{O}_{3}$ & 8.55 & 0.01 & 0.16 & 10 & 2.02 & 0.02 & 1.04 & 5 & 7.05 & 0.02 & 0.23 & 5 \\
\hline $\mathrm{MnO}$ & 0.14 & 0 & 0.96 & 10 & 0.01 & 0 & 15.38 & 5 & 0.14 & 0 & 0.71 & 5 \\
\hline $\mathrm{MgO}$ & 7.66 & 0.03 & 0.34 & 10 & 0.09 & 0.01 & 13.33 & 5 & 1.61 & 0.02 & 0.99 & 5 \\
\hline $\mathrm{CaO}$ & 11.13 & 0.02 & 0.21 & 10 & 0.75 & 0 & 0.4 & 5 & 5.68 & 0.03 & 0.48 & 5 \\
\hline $\mathrm{Na}_{2} \mathrm{O}$ & 3.12 & 0.01 & 0.4 & 10 & 2.91 & 0.01 & 0.34 & 5 & 3.3 & 0.03 & 0.88 & 5 \\
\hline $\mathrm{K}_{2} \mathrm{O}$ & 0.37 & 0 & 0.32 & 10 & 4.94 & 0.01 & 0.24 & 5 & 0.75 & 0 & 0.4 & 5 \\
\hline $\mathrm{P}_{2} \mathrm{O}_{5}$ & 0.16 & 0 & 0.92 & 10 & 0.01 & 0 & 6 & 5 & 0.17 & 0 & 1.21 & 5 \\
\hline LOI 1 & 0.73 & & & 1 & 0.63 & & & 1 & 0.38 & & & 1 \\
\hline LOI 2 & & & & & 0.59 & & & 1 & 0.34 & & & 1 \\
\hline
\end{tabular}

Note: Analyses for BOB-1 were repeated on the same fusion disks and therefore reflect instrumental precision. Analyses for NIM-G, JA-1, SO-1, and SO-2 were performed on three separate fusion disks and reflect the precision of the sample preparation procedures and the instrumental precision. The analyses are reported on a fully oxidized, volatile-free basis.

Table 3. Analytical conditions for trace-element determinations on the University of Leicester Department of Geology Philips PW1400 and ARL8420+.

\begin{tabular}{|c|c|c|c|c|c|c|c|c|c|c|}
\hline Element & Line & Crystal & $\begin{array}{l}\text { Peak angle } \\
\text { (s) }\end{array}$ & $\begin{array}{l}\text { Count time } \\
\text { angle }\end{array}$ & $\begin{array}{l}\text { Background } 1 \\
\text { (s) }\end{array}$ & $\begin{array}{l}\text { Count time } \\
\text { angle }\end{array}$ & $\begin{array}{l}\text { Background } 2 \\
\text { (s) }\end{array}$ & Count time & Collimator & Detector \\
\hline $\mathrm{Nb}$ & $\mathrm{Kal}, 2$ & $\mathrm{LiF} 220$ & 30.43 & 100 & 33.025 & 50 & 29.83 & 50 & Fine Scint. & \\
\hline $\mathrm{Zr}$ & $\mathrm{Kal}, 2$ & LiF220 & 32.115 & 80 & 33.025 & 50 & 29.83 & 50 & Fine Scint. & \\
\hline $\mathrm{Y}$ & $\mathrm{Kal} .2$ & $\mathrm{LiF} 220$ & 33.925 & 80 & 33.025 & 50 & 29.83 & 50 & Fine Scint. & \\
\hline $\mathrm{Sr}$ & $\mathrm{Kal}, 2$ & $\mathrm{LiF} 220$ & 35.88 & 80 & 36.58 & 50 & 33.025 & 50 & Fine Scint. & \\
\hline $\mathrm{Rb}$ & $\mathrm{Kal}, 2$ & LiF220 & 38.045 & 80 & 38.7 & 50 & 36.58 & 50 & Fine Scint. & \\
\hline $\mathrm{Th}$ & Lal & $\mathrm{LiF} 220$ & 39.3 & 100 & 41.015 & 50 & 38.7 & 50 & Fine Scint. & \\
\hline $\mathrm{Ga}$ & $\mathrm{Ka} 1,2$ & LiF200 & 38.965 & 40 & 39.565 & 20 & 38.265 & 20 & Fine & $\mathrm{FPC}+$ Scint \\
\hline $\mathrm{Zn}$ & $\mathrm{Kal}, 2$ & $\mathrm{LiF} 200$ & 41.805 & 40 & 42.605 & 20 & 39.565 & 20 & Fine & $\mathrm{FPC}+$ Scint \\
\hline $\mathrm{Ni}$ & $\mathrm{Kal}, 2$ & LiF200 & 48.625 & 40 & 49.885 & 20 & 47.125 & 20 & Fine & FPC + Scint. \\
\hline $\mathrm{Ce}$ & Lbl & $\mathrm{LiF} 220$ & 111.64 & 160 & 114.07 & 160 & 110.14 & 160 & Std. Coarse & FPC \\
\hline Nd & Lal & LiF220 & 112.67 & 160 & 114.07 & 160 & 110.14 & 160 & Std. Coarse & FPC \\
\hline $\mathrm{La}$ & Lal & $\mathrm{LiF} 220$ & 138.78 & 160 & 141.78 & 80 & 136.78 & 80 & Std. Coarse & FPC \\
\hline $\mathrm{Ba}$ & Lal & $\mathrm{LiF} 220$ & 154.17 & 100 & & & 153.17 & 100 & Std. Coarse & FPC \\
\hline $\mathrm{Fe}$ & $\mathrm{Kal}, 2$ & LiF200 & 57.52 & 25 & 60.42 & 10 & 55.25 & 10 & Fine & FPC \\
\hline $\mathrm{Ti}$ & $\mathrm{Kal}, 2$ & LiF200 & 86.14 & 40 & 88.8 & 15 & 84.25 & 15 & Fine & FPC \\
\hline Co & $\mathrm{Kal}, 2$ & LiF200 & 77.83 & 100 & 81.03 & 40 & 74.03 & 40 & Fine & FPC \\
\hline $\mathrm{Cr}$ & $\mathrm{Kal}, 2$ & $\mathrm{LiF} 200$ & 107.02 & 100 & 109.81 & 40 & 104.61 & 40 & Fine & FPC \\
\hline $\mathrm{V}$ & $\mathrm{Ka} 1,2$ & $\mathrm{LiF} 200$ & 123.17 & 100 & 126.37 & 40 & 121.17 & 40 & Fine & FPC \\
\hline $\mathrm{Sc}$ & $\mathrm{Kal}, 2$ & LiF200 & 97.7 & 100 & & & 95.9 & 100 & Fine & FPC \\
\hline $\mathrm{Ca}$ & $\mathrm{Kal}, 2$ & LiF200 & 113.09 & 25 & 115.1 & 15 & 110.1 & 15 & Fine & FPC \\
\hline $\mathrm{Cu}$ & $\mathrm{Kal} .2$ & LiF200 & 45.03 & 60 & 45.63 & 30 & 44.43 & 30 & Fine & FPC \\
\hline
\end{tabular}


Table 2 (continued).

\begin{tabular}{|c|c|c|c|c|c|c|c|}
\hline \multicolumn{4}{|c|}{ JA-1 } & \multicolumn{4}{|c|}{ SO-1SO-2 } \\
\hline Mean & Std. dev. & \%Std. dev. & $\begin{array}{c}\text { Number } \\
\text { of runs }\end{array}$ & Mean & Std. dev. & \%Std. dev. & $\begin{array}{c}\text { Number } \\
\text { of runs }\end{array}$ \\
\hline 59.09 & 0.53 & 0.9 & 5 & 61.47 & 0.39 & 0.64 & 5 \\
\hline 0.91 & 0.01 & 0.66 & 5 & 1.61 & 0.01 & 0.37 & 5 \\
\hline 18.13 & 0.1 & 0.56 & 5 & 16.15 & 0.1 & 0.62 & 5 \\
\hline 9.04 & 0.01 & 0.14 & 5 & 8.96 & 0.03 & 0.37 & 5 \\
\hline 0.12 & 0 & 1.67 & 5 & 0.01 & 0 & 10 & 5 \\
\hline 4.1 & 0.03 & 0.76 & 5 & 1.05 & 0.02 & 1.81 & 5 \\
\hline 2.62 & 0.01 & 0.57 & 5 & 3.01 & 0.01 & 0.02 & 5 \\
\hline 2.34 & 0.02 & 0.9 & 5 & 2.36 & 0.02 & 0.97 & 5 \\
\hline 3.28 & 0.02 & 0.64 & 5 & 3.23 & 0.01 & 0.28 & 5 \\
\hline 0.16 & 0 & 1.25 & 5 & 0.79 & 0.01 & 0.76 & 5 \\
\hline 3.9 & & & 1 & 11.07 & & & 1 \\
\hline 4.02 & & & 1 & 11.42 & & & 1 \\
\hline
\end{tabular}

Table 4. Mean values and I-sigma standard deviations for the trace elements determined on the PW1400 and the ARL8420+ from a selection of reference materials analyzed with the Site 808 Samples.

\begin{tabular}{|c|c|c|c|c|c|c|c|c|c|c|c|c|c|c|c|c|}
\hline \multirow[b]{2}{*}{ Element } & \multicolumn{4}{|c|}{ Reference Sample } & \multicolumn{4}{|c|}{ NIM-G } & \multicolumn{4}{|c|}{ JA-1 } & \multicolumn{4}{|c|}{ SO-1 SO-2 } \\
\hline & Mean & Std. dev. & \%Std. dev. & $\begin{array}{c}\text { Number } \\
\text { of runs }\end{array}$ & Mean & Std. dev. & \%Std. Dev. & $\begin{array}{l}\text { Number } \\
\text { of runs }\end{array}$ & Mean & Std. dev. & \%Std. dev. & $\begin{array}{l}\text { Number } \\
\text { of runs }\end{array}$ & Mean & Std. dev. & \%Std. dev. & $\begin{array}{l}\text { Number } \\
\text { of runs }\end{array}$ \\
\hline $\mathrm{Nb}$ & 51.51 & 3.45 & 6.7 & 8 & 7.14 & 2.42 & 33.88 & 5 & 16.6 & 2.86 & 17.22 & 10 & 30.34 & 2.63 & 8.67 & 8 \\
\hline $\mathrm{Zr}$ & 271.57 & 9.46 & 3.48 & 8 & 85.1 & 2.52 & 2.96 & 5 & 98.18 & 3.7 & 3.77 & 10 & 953.28 & 63.62 & 6.67 & 8 \\
\hline$\gamma$ & 152.4 & 5.56 & 3.65 & 8 & 32.2 & 2.82 & 8.8 & 5 & 26.55 & 3.86 & 14.54 & 10 & 54.09 & 4.75 & 8.79 & 8 \\
\hline $\mathrm{Sr}$ & 11.32 & 1.27 & 11.25 & 8 & 249.68 & 1.92 & 0.77 & 5 & 330.92 & 9.85 & 2.98 & 10 & 356.99 & 19.35 & 5.42 & 8 \\
\hline $\mathbf{R b}$ & 324.69 & 8.6 & 2.65 & 8 & 6.3 & 1.25 & 19.9 & 5 & 142.97 & 7.12 & 4.98 & 10 & 78.59 & 8.49 & 10.81 & 8 \\
\hline Th & 50.47 & 3.31 & 6.57 & 8 & 3.5 & 2.73 & 78.08 & 5 & 19.43 & 3.53 & 18.16 & 10 & 6.2 & 4.44 & 71.53 & 8 \\
\hline Ga & 22.96 & 2.81 & 12.25 & 8 & 19.86 & 1.21 & 6.11 & 5 & 19.96 & 1.7 & 8.51 & 10 & 24.25 & 1.68 & 6.92 & 8 \\
\hline $\mathrm{Zn}$ & 62.3 & 1.34 & 2.15 & 8 & 108.8 & 2.03 & 1.86 & 5 & 181.11 & 21.16 & 11.69 & 10 & 141.25 & 4.56 & 3.23 & 8 \\
\hline $\mathrm{Ni}$ & 0.19 & 0.23 & 120.55 & 8 & 0.72 & 0.9 & 125.28 & 5 & 97.39 & 9.62 & 9.88 & 10 & 4.49 & 2.96 & 65.89 & 8 \\
\hline $\mathrm{Sc}$ & 3.27 & 2.25 & 68.79 & 7 & 26.3 & 1.99 & 7.56 & 4 & 18.04 & 2.43 & 13.48 & 7 & 12.34 & 2.82 & 22.87 & 7 \\
\hline v & 1.21 & 1.67 & 137.69 & 7 & 99.45 & 2.85 & 2.87 & 4 & 133.21 & 7.57 & 5.68 & 7 & 64.26 & 6.61 & 10.28 & 7 \\
\hline $\mathrm{Cr}$ & 14.16 & 4.49 & 31.7 & 7 & 10.2 & 3.99 & 39.16 & 4 & 173.83 & 4.65 & 2.67 & 7 & 13.8 & 2.37 & 17.14 & 7 \\
\hline Co & \multicolumn{3}{|c|}{ not detectable } & 7 & 17.28 & 1.54 & 8.89 & 4 & 34.16 & 1.9 & 5.56 & 7 & 18 & 1.95 & 10.81 & 7 \\
\hline $\mathrm{Cu}$ & 5.99 & 1.25 & 20.86 & 7 & 40.45 & 2.55 & 6.3 & 4 & 62.39 & 2.94 & 4.72 & 7 & 8.2 & 2.64 & 32.22 & 7 \\
\hline Ba & 107.04 & 3.34 & 3.12 & 7 & 303.42 & 4.57 & 1.51 & 4 & 949.51 & 12.04 & 1.27 & 7 & 1153.07 & 30.47 & 2.64 & 7 \\
\hline $\mathrm{La}$ & 104.84 & 1.34 & 1.28 & 7 & 4.17 & 2.29 & 54.84 & 4 & 53.53 & 2.11 & 3.94 & 7 & 44.71 & 2.72 & 6.09 & 7 \\
\hline $\mathrm{Ce}$ & 192.79 & 7.25 & 3.76 & 7 & 11.93 & 7.24 & 60.7 & 4 & 100.13 & 5.5 & 5.5 & 7 & 114.71 & 7.04 & 6.14 & 7 \\
\hline $\mathrm{Nd}$ & 68.91 & 3.35 & 4.86 & 7 & 9.53 & 1.64 & 17.24 & 4 & 42.61 & 4.16 & 9.77 & 7 & 55.37 & 3.99 & 7.21 & 7 \\
\hline
\end{tabular}

Note: Analyses were repeated on the same fusion disks as the major-element determinations and therefore reflect sample preparation and instrumental precision. Note the relatively high standard deviation on the Zr determinations for SO-2. This may reflect a degree of sample inhomogeneity with respect to $\mathrm{Zr}$ distribution, perhaps a result of accessory phase control of the $\mathrm{Zr}$ content. This problem is not so apparent with the large samples analyzed by conventional powder pellet techniques. The analyses are reported on a fully oxidized, volatile-free basis. 
Table 5. XRF analyses of samples from Site 808. Analyses recalculated to include loss on ignition (LOI).

\begin{tabular}{|c|c|c|c|c|c|c|c|c|}
\hline Sample & $\begin{array}{l}\text { Hole, core, section, } \\
\text { interval }(\mathrm{cm})\end{array}$ & Depth (mbsf) & $\mathrm{SiO}_{2}$ & $\mathrm{TiO}_{2}$ & $\mathrm{Al}_{2} \mathrm{O}_{3}$ & $\mathrm{Fe}_{2} \mathrm{O}_{3}$ & $\mathrm{MnO}$ & $\mathrm{MgO}$ \\
\hline 19 & $808 \mathrm{~A}-1 \mathrm{H}-3,42-44$ & 3.40 & 61.00 & 0.629 & 15.65 & 5.81 & 0.084 & 2.50 \\
\hline 31 & 808A-IH-3-A. $65-67$ & 3.65 & 60.50 & 0.592 & 14.60 & 5.22 & 0.074 & 2.35 \\
\hline 43 & $808 \mathrm{~A}-1 \mathrm{H}-3-\mathrm{B}, 65-67$ & 3.65 & 60.30 & 0.594 & 14.19 & 5.18 & 0.074 & 2.40 \\
\hline 76 & $808 \mathrm{~A}-2 \mathrm{H}-1,24-26$ & 6.57 & 60.71 & 0.654 & 15.59 & 5.56 & 0.075 & 2.41 \\
\hline 145 & $808 \mathrm{~A}-2 \mathrm{H}-1,40-42$ & 6.71 & 60.90 & 0.722 & 15.34 & 5.92 & 0.083 & 2.57 \\
\hline 146 & $808 \mathrm{~A}-2 \mathrm{H}-1,49-51$ & 6.80 & 66.64 & 0.634 & 13.76 & 4.82 & 0.067 & 2.11 \\
\hline 166 & $808 \mathrm{~A}-2 \mathrm{H}-1,54-56$ & 6.84 & 59.74 & 0.713 & 15.00 & 5.69 & 0.068 & 2.50 \\
\hline 8 & $808 \mathrm{~A}-2 \mathrm{H}-3,55-57$ & 9.85 & 58.34 & 0.647 & 15.21 & 5.47 & 0.083 & 2.59 \\
\hline 214 & $808 \mathrm{~A}-3 \mathrm{H}-1,80-82$ & 16.60 & 60.95 & 0.696 & 14.88 & 5.59 & 0.072 & 2.35 \\
\hline 7 & $808 \mathrm{~A}-3 \mathrm{H}-3,79-81$ & 19.49 & 60.40 & 0.683 & 15.58 & 5.49 & 0.075 & 2.41 \\
\hline 6 & $808 \mathrm{~A}-3 \mathrm{H}-3,142-144$ & 20.12 & 61.43 & 0.653 & 15.46 & 5.61 & 0.085 & 2.38 \\
\hline 212 & $808 \mathrm{~A}-4 \mathrm{H}-1,38-40$ & 25.68 & 56.57 & 0.658 & 14.68 & 5.82 & 0.070 & 2.43 \\
\hline 118 & $808 \mathrm{~A}-4 \mathrm{H}-3,39-41$ & 28.22 & 59.39 & 0.714 & 15.75 & 6.00 & 0.074 & 2.58 \\
\hline 80 & $808 \mathrm{~A}-5 \mathrm{H}-1,45-47$ & 35.25 & 60.32 & 0.659 & 16.01 & 6.20 & 0.074 & 2.61 \\
\hline 38 & $808 \mathrm{~A}-6 \mathrm{H}-1,89-91$ & 45.19 & 59.10 & 0.707 & 16.56 & 6.55 & 0.104 & 2.69 \\
\hline 149 & $808 \mathrm{~A}-7 \mathrm{H}-2.20-22$ & 55.50 & 59.66 & 0.716 & 16.02 & 6.49 & 0.088 & 2.66 \\
\hline 144 & $808 \mathrm{~A}-7 \mathrm{H}-4,85-87$ & 59.10 & 61.62 & 0.703 & 15.41 & 5.77 & 0.067 & 2.42 \\
\hline 34 & $808 \mathrm{~A}-8 \mathrm{H}-1-\mathrm{A} .90-92$ & 64.20 & 65.29 & 0.615 & 14.84 & 5.80 & 0.098 & 2,69 \\
\hline 41 & $808 \mathrm{~A}-8 \mathrm{H}-1-\mathrm{B}, 90-92$ & 64.20 & 65.72 & 0.616 & 14.73 & 5.85 & 0.098 & 2.72 \\
\hline 20 & $808 \mathrm{~A}-8 \mathrm{H}-1-\mathrm{A}, 117-119$ & 64.48 & 62.83 & 0.721 & 15.67 & 5.45 & 0.104 & 2.34 \\
\hline 37 & $808 \mathrm{~A}-8 \mathrm{H}-1-\mathrm{B}, 117-119$ & 64.48 & 63.42 & 0.691 & 14.80 & 5.22 & 0.091 & 2.26 \\
\hline 114 & $808 \mathrm{~A}-9 \mathrm{H}-1,33-35$ & 68.65 & 59.40 & 0.671 & 15.59 & 6.43 & 0.095 & 2.63 \\
\hline 147 & $808 \mathrm{~A}-9 \mathrm{H}-4,106-108$ & 73.86 & 60.54 & 0.754 & 16.37 & 6.30 & 0.092 & 2.71 \\
\hline 119 & $808 \mathrm{~A}-10 \mathrm{H}-1,1-3$ & 77.81 & 60.61 & 0.748 & 16.11 & 6.10 & 0.061 & 2.67 \\
\hline 74 & $808 \mathrm{~A}-10 \mathrm{H}-1,31-33$ & 78.11 & 63.22 & 0.649 & 15.34 & 5.59 & 0.076 & 2.37 \\
\hline 93 & $808 B-2 X-1.62-64$ & 121.22 & 62.74 & 0.685 & 15.19 & 5.21 & 0.068 & 2.18 \\
\hline 35 & 808B-2X-1, 68-70 & 121.28 & 67.54 & 0.536 & 13.98 & 3.56 & 0.086 & 1.28 \\
\hline 83 & $808 \mathrm{~B}-4 \mathrm{X}-1,50-52$ & 140.40 & 66.81 & 0.612 & 13.58 & 4.92 & 0.057 & 2.31 \\
\hline 171 & $808 \mathrm{~B}-4 \mathrm{X}-1,69-71$ & 140.59 & 63.37 & 0.686 & 14.68 & 5.78 & 0.071 & 2.46 \\
\hline 117 & $808 \mathrm{~B}-5 \mathrm{X}-1.46-48$ & 149.96 & 61.23 & 0.712 & 15.53 & 6.44 & 0.064 & 2.52 \\
\hline 21 & $808 B-5 X-2-A, 14-16$ & 150.67 & 59.20 & 0.727 & 16.23 & 6.81 & 0.113 & 2.77 \\
\hline 32 & 808B-5X-2-B. $14-16$ & 150.67 & 59.10 & 0.705 & 16.01 & 6.64 & 0.113 & 2.73 \\
\hline 86 & $808 \mathrm{~B}-9 \mathrm{X}-1.67-69$ & 188.87 & 60.61 & 0.677 & 14.85 & 5.69 & 0.089 & 2.43 \\
\hline 75 & $808 \mathrm{~B}-10 \mathrm{X}-1.74-76$ & 198.14 & 64.76 & 0.606 & 14.33 & 5.15 & 0.075 & 1.85 \\
\hline 170 & $808 B-10 X-3,39-41$ & 200.79 & 67.09 & 0.631 & 13.80 & 4.25 & 0.061 & 1.89 \\
\hline 16 & $808 \mathrm{~B}-11 \mathrm{X}-1-\mathrm{A}, 91-93$ & 207.71 & 62.61 & 0.661 & 15.34 & 5.32 & 0.094 & 2.29 \\
\hline 36 & $808 \mathrm{~B}-11 \mathrm{X}-1-\mathrm{B}, 91-93$ & 207.71 & 63.02 & 0.680 & 15.24 & 5.23 & 0.094 & 2.30 \\
\hline 84 & $808 \mathrm{~B}-11 \mathrm{X}-1,112-114$ & 207.93 & 62.58 & 0.633 & 13.87 & 5.08 & 0.079 & 2.10 \\
\hline 148 & $808 \mathrm{~B}-13 \mathrm{X}-1.45-47$ & 226.25 & 66.22 & 0.671 & 13.69 & 4.76 & 0.066 & 2.10 \\
\hline 78 & $808 \mathrm{~B}-13 \mathrm{X}-1,80-82$ & 226.60 & 62.77 & 0.689 & 15.53 & 5.37 & 0.093 & 2.30 \\
\hline 85 & $808 \mathrm{~B}-14 \mathrm{X}-1.9-11$ & 235.19 & 60.77 & 0.694 & 14.54 & 5.92 & 0.087 & 2.36 \\
\hline 91 & $808 \mathrm{~B}-15 \mathrm{X}-1,1-3$ & 244.51 & 60.00 & 0.657 & 14.67 & 6.06 & 0.073 & 2.48 \\
\hline 22 & 808B-16X-1-A. $33-35$ & 254.53 & 62.74 & 0.666 & 15.00 & 5.36 & 0.086 & 2.19 \\
\hline 29 & 808 B-16X-1-B, $33-35$ & 254.53 & 63.87 & 0.675 & 15.22 & 5.50 & 0.086 & 2.20 \\
\hline 108 & 808 B- 16 X-1. $72-74$ & 254.92 & 59.49 & 0.722 & 15.31 & 6.54 & 0.087 & 2.50 \\
\hline 213 & $808 \mathrm{~B}-17 \mathrm{X}-1,134-136$ & 264.74 & 60.04 & 0.695 & 15.00 & 6.49 & 0.165 & 2.64 \\
\hline 112 & $808 B-17 X-2,95-97$ & 265.85 & 56.40 & 0.728 & 16.02 & 7.28 & 0.093 & 3.09 \\
\hline 128 & $808 \mathrm{~B}-19 \mathrm{X}-2,144-146$ & 285.46 & 61.47 & 0.667 & 15.19 & 6.59 & 0.107 & 2.53 \\
\hline 143 & 808B-19X-3, $16-18$ & 285.66 & 64.49 & 0.670 & 14.46 & 5.14 & 0.069 & 2.18 \\
\hline 115 & $808 B-20 X-C C, 3-5$ & 297.24 & 66.96 & 0.626 & 14.28 & 4.62 & 0.055 & 2.01 \\
\hline 162 & $808 \mathrm{~B}-20 \mathrm{X}-\mathrm{CC}, 6-8$ & 297.27 & 60.24 & 0.724 & 14.80 & 6.23 & 0.089 & 2.38 \\
\hline 123 & $808 \mathrm{C}-1 \mathrm{R}-2,0-2$ & 300.01 & 60.38 & 0.712 & 14.74 & 5.87 & 0.100 & 2.39 \\
\hline 172 & $808 C-1 R-2,17-19$ & 300.18 & 57.97 & 0.553 & 11.50 & 6.48 & 0.172 & 1.75 \\
\hline 168 & $808 C-1 R-2,47-50$ & 300.48 & 59.64 & 0.680 & 15.74 & 7.13 & 0.192 & 2.40 \\
\hline 184 & $808 \mathrm{~B}-21 \mathrm{X}-\mathrm{CC} .37-39$ & 307.06 & 61.08 & 0.772 & 15.61 & 6.41 & 0.097 & 2.55 \\
\hline 13 & $808 \mathrm{~B}-22 \mathrm{X} \cdot 1-\mathrm{A} \cdot 65-67$ & 307.77 & 60.05 & 0.694 & 16.04 & 6.29 & 0.112 & 2.62 \\
\hline 24 & 808 B-22X-1-B, $65-67$ & 307.77 & 60.33 & 0.680 & 15.53 & 6.20 & 0.106 & 2.59 \\
\hline 165 & $808 B-22 X-2,6-8$ & 308.68 & 65.92 & 0.654 & 14.17 & 4.77 & 0.067 & 2.05 \\
\hline 124 & $808 \mathrm{~B}-23 \mathrm{X}-1,43-45$ & 317.24 & 63.12 & 0.743 & 16.50 & 5.75 & 0.064 & 2.35 \\
\hline 132 & $808 \mathrm{C}-3 \mathrm{R}-1,20-22$ & 317.81 & 61.09 & 0.715 & 15.85 & 6.45 & 0.095 & 2.71 \\
\hline 204 & $808 C-3 R-1,40-42$ & 318.01 & 61.13 & 0.703 & 15.08 & 5.84 & 0.080 & 2.44 \\
\hline 101 & $808 \mathrm{C}-4 \mathrm{R}-1,41-42$ & 327.61 & 61.01 & 0.708 & 15.22 & 6.10 & 0.077 & 2.45 \\
\hline 59 & $808 \mathrm{C}-4 \mathrm{R}-1,99-102$ & 328.20 & 61.69 & 0.708 & 16.62 & 6.27 & 0.075 & 2.60 \\
\hline 120 & $808 \mathrm{~B}-24 \mathrm{X}-2,59-61$ & 328.51 & 61.02 & 0.720 & 15.76 & 6.03 & 0.084 & 2.51 \\
\hline 137 & $808 B-24 X-2,70-85$ & 328.63 & 61.93 & 0.708 & 15.06 & 6.47 & 0.082 & 2.46 \\
\hline 99 & $808 C-4 R-2,47-49$ & 329.18 & 59.03 & 0.676 & 14.93 & 5.54 & 0.097 & 2.39 \\
\hline 125 & $808 \mathrm{~B}-24 \mathrm{X}-\mathrm{CC}, 5-7$ & 335.71 & 62.19 & 0.692 & 14.96 & 6.05 & 0.098 & 2.40 \\
\hline 142 & $808 \mathrm{~B}-25 \mathrm{X}-1,99-100$ & 336.99 & 60.46 & 0.707 & 15.53 & 6.36 & 0.089 & 2.70 \\
\hline 113 & $808 B-25 X-2,23-24$ & 337.73 & 66.13 & 0.673 & 12.40 & 5.12 & 0.192 & 2.04 \\
\hline 116 & $808 \mathrm{~B}-25 \mathrm{X}-2,59-60$ & 338.09 & 60.74 & 0.714 & 15.09 & 6.13 & 0.090 & 2.55 \\
\hline 18 & $808 \mathrm{~B}-25 \mathrm{X}-2-\mathrm{A}, 135-150$ & 338.92 & 59.51 & 0.676 & 16.15 & 6.66 & 0.113 & 2.69 \\
\hline 28 & $808 B-25 X-2-B, 135-150$ & 338.92 & 59.66 & 0.683 & 16.03 & 6.61 & 0.112 & 2.72 \\
\hline 90 & $808 \mathrm{~B}-25 \mathrm{X}-3,44-49$ & 339.45 & 59.64 & 0.696 & 15.07 & 6.40 & 0.096 & 2.59 \\
\hline 87 & $808 \mathrm{~B}-25 \mathrm{X}-3,80-81$ & 339.81 & 60.34 & 0.701 & 15.24 & 6.35 & 0.089 & 2.59 \\
\hline 17 & $808 \mathrm{~B}-25 \mathrm{X}-4-\mathrm{A}, 20-21$ & 340.71 & 60.01 & 0.687 & 16.10 & 6.55 & 0.113 & 2.65 \\
\hline 27 & 808 B-25X-4-B, $20-21$ & 340.71 & 60.17 & 0.685 & 16.11 & 6.59 & 0.113 & 2.65 \\
\hline 26 & $808 \mathrm{~B}-25 \mathrm{X}-4-\mathrm{B}, 89-90$ & 341.40 & 60.29 & 0.685 & 16.15 & 6.33 & 0.094 & 2.66 \\
\hline 136 & 808B-25X-4-A. 89-90 & 341.40 & 60.83 & 0.674 & 15.13 & 6.20 & 0.087 & 2.62 \\
\hline 121 & $808 C-6 R-2,98-99$ & 348.56 & 61.90 & 0.735 & 16.11 & 6.17 & 0.064 & 2.62 \\
\hline 174 & $808 C-6 R-3.54-55$ & 350.14 & 62.85 & 0.726 & 15.91 & 6.26 & 0.080 & 2.47 \\
\hline 14 & 808 C-6R-3-A. $76-77$ & 350.36 & 61.88 & 0.698 & 16.72 & 6.10 & 0.124 & 2.42 \\
\hline 42 & $808 C-6 R-3-B, 76-77$ & 350.36 & 62.11 & 0.707 & 16.40 & 6.15 & 0.124 & 2.47 \\
\hline 152 & $808 \mathrm{C}-7 \mathrm{R}-1,33-34$ & 356.03 & 61.87 & 0.738 & 15.33 & 6.18 & 0.070 & 2.46 \\
\hline 88 & $808 C-8 R-1,51-53$ & 366.42 & 60.77 & 0.689 & 15.23 & 5.94 & 0.086 & 2.41 \\
\hline 159 & $808 C-8 R-1,131-133$ & 367.22 & 59.47 & 0.715 & 14.96 & 6.50 & 0.078 & 2.63 \\
\hline 100 & $808 C-10 R-2,105-106$ & 387.75 & 59.83 & 0.705 & 15.08 & 6.37 & 0.082 & 2.67 \\
\hline 134 & $808 \mathrm{C}-1 / \mathrm{R}-1,108-109$ & 395.88 & 62.91 & 0.710 & 15.12 & 6.02 & 0.075 & 2.56 \\
\hline 153 & $808 \mathrm{C}-12 \mathrm{R}-3,24-26$ & 407.85 & 60.87 & 0.770 & 15.39 & 6.14 & 0.107 & 2.57 \\
\hline 179 & $808 C-12 R-3,140-142$ & 407.51 & 59.98 & 0.682 & 15.38 & 6.52 & 0.103 & 2.62 \\
\hline
\end{tabular}


Table 5 (continued).

\begin{tabular}{|c|c|c|c|c|c|c|c|c|}
\hline Sample & $\begin{array}{l}\text { Hole, core, section, } \\
\text { interval }(\mathrm{cm})\end{array}$ & $\mathrm{Nb}$ & $\mathrm{Zr}$ & $\mathbf{Y}$ & Sr & $\mathrm{Rb}$ & Ga & $\mathrm{Zn}$ \\
\hline 19 & $808 \mathrm{~A}-1 \mathrm{H}-3,42-44$ & 9.5 & 142.0 & 27.9 & 136.0 & 107.0 & 18.2 & 66.4 \\
\hline 31 & 808 A-1H-3-A, $65-67$ & 13.5 & 164.4 & 37.2 & 175.4 & 91.4 & 16.8 & 32.4 \\
\hline 43 & $808 \mathrm{~A}-1 \mathrm{H}-3-\mathrm{B}, 65-67$ & 4.9 & 170.7 & 31.9 & 190.1 & 100.8 & 17.3 & 40.3 \\
\hline 76 & $808 \mathrm{~A}-2 \mathrm{H}-1.24-26$ & 10.8 & 149.9 & 23.2 & 142.0 & 97.6 & 20.0 & 83.4 \\
\hline 145 & $808 \mathrm{~A}-2 \mathrm{H}-1,40-42$ & 17.4 & 187.8 & 36.8 & 165.5 & 139.4 & 22.5 & 61.0 \\
\hline 146 & $808 \mathrm{~A}-2 \mathrm{H}-1,49-51$ & 10.4 & 238.1 & 34.0 & 183.5 & 99.9 & 21.0 & 58.6 \\
\hline 166 & $808 \mathrm{~A}-2 \mathrm{H}-1.54-56$ & 17.3 & 186.4 & 44.7 & 169.5 & 131.3 & 20.2 & 61.7 \\
\hline 8 & $808 \mathrm{~A}-2 \mathrm{H}-3,55-57$ & 12.1 & 155.5 & 24.6 & 184.5 & 104.8 & 15.2 & 54.2 \\
\hline 214 & $808 \mathrm{~A}-3 \mathrm{H}-1,80-82$ & 11.0 & 189.9 & 33.8 & 165.0 & 105.8 & 25.0 & 63.5 \\
\hline 7 & $808 \mathrm{~A}-3 \mathrm{H}-3,79-81$ & 20.8 & 180.8 & 33.2 & 161.2 & 118.3 & 19.8 & 61.6 \\
\hline 6 & $808 \mathrm{~A}-3 \mathrm{H}-3,142-144$ & 9.2 & 173.1 & 25.3 & 177.6 & 98.4 & 18.3 & 71.8 \\
\hline 212 & $808 \mathrm{~A}-4 \mathrm{H}-1.38-40$ & 10.2 & 135.0 & 35.3 & 242.3 & 95.3 & 20.7 & 46.5 \\
\hline 118 & $808 \mathrm{~A}-4 \mathrm{H}-3,39-41$ & 10.9 & 145.5 & 32.5 & 191.9 & 94.6 & 16.5 & 53.6 \\
\hline 80 & $808 \mathrm{~A}-5 \mathrm{H}-1,45-47$ & 15.0 & 164.4 & 32.1 & 167.8 & 119.7 & 21.6 & 97.1 \\
\hline 38 & $808 \mathrm{~A}-6 \mathrm{H}-1,89-91$ & 6.6 & 150.0 & 30.8 & 184.7 & 111.0 & 16.9 & 65.7 \\
\hline 149 & $808 \mathrm{~A}-7 \mathrm{H}-2,20-22$ & 11.2 & 170.1 & 36.5 & 213.8 & 124.5 & 22.2 & 71.1 \\
\hline 144 & $808 \mathrm{~A}-7 \mathrm{H}-4,85-87$ & 14.7 & 159.4 & 22.3 & 181.1 & 110.7 & 16.9 & 66.9 \\
\hline 34 & 808 A-8H-1-A, $90-92$ & 8.8 & 118.3 & 20.3 & 229.0 & 62.9 & 17.4 & 58.5 \\
\hline 41 & $808 \mathrm{~A}-8 \mathrm{H}-1-\mathrm{B}, 90-92$ & 4.6 & 110.1 & 16.8 & 225.3 & 60.6 & 14.7 & 57.9 \\
\hline 20 & $808 \mathrm{~A}-8 \mathrm{H}-1-\mathrm{A}, 117-119$ & 11.9 & 217.0 & 40.6 & 142.2 & 117.4 & 22.3 & 82.2 \\
\hline 37 & $808 \mathrm{~A}-8 \mathrm{H}-1-\mathrm{B}, 117-119$ & 8.3 & 195.8 & 32.9 & 131.4 & 106.4 & 15.7 & 69.1 \\
\hline 114 & $808 \mathrm{~A}-9 \mathrm{H}-1,33-35$ & 14.0 & 154.8 & 34.4 & 170.9 & 110.7 & 18.1 & 71.8 \\
\hline 147 & $808 \mathrm{~A}-9 \mathrm{H}-4,106-108$ & 7.7 & 166.6 & 32.6 & 167.7 & 137.9 & 23.9 & 73.5 \\
\hline 119 & $808 \mathrm{~A}-10 \mathrm{H}-1, \mathrm{I}-3$ & 14.0 & 152.6 & 29.6 & 147.1 & 117.8 & 20.8 & 81.3 \\
\hline 74 & $808 \mathrm{~A}-10 \mathrm{H}-1.31-33$ & 6.3 & 159.2 & 22.8 & 210.8 & 84.7 & 19.5 & 79.6 \\
\hline 93 & $808 \mathrm{~B}-2 \mathrm{X}-1.62-64$ & 12.3 & 159.9 & 28.4 & 140.7 & 103.1 & 21.6 & 81.4 \\
\hline 35 & $808 B-2 X-1,68-70$ & 8.7 & 186.7 & 40.1 & 131.3 & 91.1 & 16.4 & 50.2 \\
\hline 83 & $808 \mathrm{~B}-4 \mathrm{X}-1.50-52$ & 11.6 & 161.8 & 30.1 & 181.0 & 127.0 & 20.9 & 95.5 \\
\hline 171 & $808 \mathrm{~B}-4 \times-1,69-71$ & 6.0 & 151.2 & 26.2 & 194.8 & 80.9 & 13.7 & 70.9 \\
\hline 117 & $808 \mathrm{~B}-5 \mathrm{X}-1,46-48$ & 10.3 & 143.8 & 28.9 & 140.6 & 106.4 & 19.7 & 71.3 \\
\hline 21 & $808 B-5 X-2-A, 14-16$ & 10.2 & 125.9 & 27.0 & 173.4 & 85.5 & 20.1 & 80.7 \\
\hline 32 & 808B-5X-2-B. 14-16 & 6.1 & 128.0 & 30.7 & 178.1 & 89.2 & 14.7 & 74.4 \\
\hline 86 & $808 \mathrm{~B}-9 \mathrm{X}-1.67-69$ & 7.1 & 120.5 & 28.1 & 165.5 & 81.0 & 19.2 & 88.8 \\
\hline 75 & $808 \mathrm{~B}-10 \mathrm{X}-1,74-76$ & 8.1 & 171.7 & 31.4 & 152.3 & 99.0 & 16.3 & 73.4 \\
\hline 170 & $808 \mathrm{~B}-10 \mathrm{X}-3,39-41$ & 11.7 & 226.1 & 30.8 & 199.1 & 80.5 & 16.5 & 58.1 \\
\hline 16 & 808B-11X-1-A. $91-93$ & 14.9 & 172.8 & 31.4 & 176.9 & 110.7 & 24.4 & 67.9 \\
\hline 36 & 808B-11X-1-B. 91-93 & 11.9 & 164.4 & 29.8 & 173.8 & 122.9 & 17.0 & 60.9 \\
\hline 84 & $808 \mathrm{~B}-11 \mathrm{X}-1.112-114$ & 15.1 & 166.9 & 28.7 & 180.1 & 110.1 & 19.4 & 58.7 \\
\hline 148 & $808 \mathrm{~B}-13 \mathrm{X}-1,45-47$ & 14.9 & 218.2 & 31.8 & 203.4 & 106.4 & 20.6 & 56.0 \\
\hline 78 & $808 \mathrm{~B}-13 \mathrm{X}-1,80-82$ & 10.2 & 167.3 & 29.0 & 145.5 & 118.1 & 15.6 & 90.3 \\
\hline 85 & $808 \mathrm{~B}-14 \mathrm{X}-1,9-11$ & 13.6 & 169.0 & 29.9 & 166.7 & 118.0 & 18.7 & 91.0 \\
\hline 91 & $808 \mathrm{~B}-15 \mathrm{X}-1,1-3$ & 9.3 & 148.7 & 32.1 & 184.3 & 101.0 & 21.4 & 1040.3 \\
\hline 22 & $808 \mathrm{~B}-16 \mathrm{X}-1-\mathrm{A}, 33-35$ & 15.1 & 204.0 & 26.2 & 165.4 & 108.3 & 21.7 & 85.7 \\
\hline 29 & $808 \mathrm{~B}-16 \mathrm{X}-1-\mathrm{B}, 33-35$ & 15.7 & 185.9 & 28.7 & I5I.1 & 98.5 & 17.7 & 66.9 \\
\hline 108 & $808 B-16 X-1,72-74$ & 11.5 & 150.0 & 26.5 & 155.4 & 120.9 & 19.2 & 85.7 \\
\hline 213 & $808 \mathrm{~B}-17 \mathrm{X}-1,134-136$ & 6.5 & 126.5 & 24.8 & 206.3 & 62.2 & 16.3 & 69.5 \\
\hline 112 & $808 \mathrm{~B}-17 \mathrm{X}-2.95-97$ & 6.2 & 125.6 & 29.6 & 202.9 & 91.4 & 21.7 & 76.9 \\
\hline 128 & $808 \mathrm{~B}-19 \mathrm{X}-2,144-146$ & 13.7 & 160.5 & 31.3 & 152.3 & 127.3 & 22.3 & 82.2 \\
\hline 143 & $808 \mathrm{~B}-19 \mathrm{X}-3,16-18$ & 12.1 & 164.6 & 26.7 & 160.4 & 89.0 & 16.1 & 70.5 \\
\hline 115 & 808B-20X-CC. 3-5 & 5.5 & 180.8 & 23.6 & 195.8 & 100.9 & 23.9 & 73.5 \\
\hline 162 & 808B-20X-CC, $6-8$ & 10.8 & 167.0 & 32.7 & 152.8 & 108.1 & 15.4 & 74.4 \\
\hline 123 & $808 \mathrm{C}-1 \mathrm{R}-2,0-2$ & 12.6 & 154.2 & 35.2 & 171.3 & 104.2 & 18.8 & 73.3 \\
\hline 172 & $808 \mathrm{C}-1 \mathrm{R}-2,17-19$ & 7.0 & 115.5 & 45.2 & 279.8 & 38.8 & 15.9 & 65.3 \\
\hline 168 & $808 \mathrm{C}-1 \mathrm{R}-2,47-50$ & 14.7 & 161.6 & 30.1 & 136.0 & 140.4 & 21.0 & 90.3 \\
\hline 184 & 808B-2IX-CC. $37-39$ & 12.1 & 181.7 & 35.8 & 128.8 & 129.6 & 18.4 & 85.5 \\
\hline 13 & $808 \mathrm{~B} \cdot 22 \mathrm{X}-1 \cdot \mathrm{A}, 65-67$ & 16,0 & 154.9 & 32.4 & 156.9 & 103.2 & 20.1 & 66.8 \\
\hline 24 & 808B-22X-1-B. $65-67$ & 14.2 & 167.1 & 44.3 & 169.3 & 117.3 & 20.5 & 74.7 \\
\hline 165 & $808 \mathrm{~B}-22 \mathrm{X}-2.6-8$ & 13.0 & 177.8 & 30.7 & 193.9 & 99.9 & 16.8 & 65.8 \\
\hline 124 & $808 \mathrm{~B}-23 \mathrm{X}-1,43-45$ & 9.6 & 176.6 & 30.1 & 169.8 & 133.2 & 20.5 & 83.0 \\
\hline 132 & $808 \mathrm{C}-3 \mathrm{R}-1.20-22$ & 15.5 & 168.3 & 32.7 & 177.9 & 135.3 & 23.7 & 84.7 \\
\hline 204 & $808 \mathrm{C}-3 \mathrm{R}-1,40-42$ & 10.7 & 162.8 & 33.1 & 185.1 & 100.3 & 18.3 & 105.4 \\
\hline 101 & $808 \mathrm{C}-4 \mathrm{R}-1,41-42$ & 8.2 & 159.5 & 31.8 & 140.6 & 112.6 & 18.0 & 78.8 \\
\hline 59 & $808 \mathrm{C}-4 \mathrm{R}-1,99-102$ & 12.0 & 158.0 & 32.9 & 135.1 & 125.2 & 19.5 & 88.8 \\
\hline 120 & $808 \mathrm{~B}-24 \mathrm{X}-2.59-61$ & 9.2 & 153.4 & 33.2 & 143.2 & 115.0 & 22.8 & 89.2 \\
\hline 137 & $808 \mathrm{~B}-24 \mathrm{X}-2,70-85$ & 10.4 & 172.4 & 32.9 & 174.3 & 114.1 & 19.4 & 81.5 \\
\hline 99 & $808 \mathrm{C}-4 \mathrm{R} \cdot 2,47-49$ & 11.2 & 136.7 & 22.5 & 153.9 & 116.7 & 22.8 & 104.4 \\
\hline 125 & $808 B-24 X-C C .5-7$ & 18.4 & 186.3 & 35.3 & 168.4 & 108.0 & 15.6 & 79.1 \\
\hline 142 & $808 \mathrm{~B}-25 \mathrm{X}-1.99-100$ & 10.1 & 160.9 & 32.2 & 148.7 & 128.7 & 21.6 & 91.3 \\
\hline 113 & $808 \mathrm{~B}-25 \mathrm{X}-2,23-24$ & 12.3 & 152,7 & 27.5 & 163.8 & 106.8 & 15.3 & 79.8 \\
\hline 116 & $808 \mathrm{~B}-25 \mathrm{X}-2,59-60$ & 12.9 & 152.7 & 29.3 & 154.7 & 109.9 & 15.6 & 87.4 \\
\hline 18 & $808 \mathrm{~B}-25 \mathrm{X}-2-\mathrm{A}, 135-150$ & 11.5 & 140.0 & 29.5 & 164.9 & 112.9 & 18.3 & 85.8 \\
\hline 28 & $808 \mathrm{~B}-25 \mathrm{X}-2-\mathrm{B} .135-150$ & 11.3 & 158.2 & 29.4 & 176.9 & 124.2 & 20.4 & 93.4 \\
\hline 90 & $808 \mathrm{~B}-25 \mathrm{X}-3.44-49$ & 15.0 & 148.9 & 26.5 & 170.0 & 108.6 & 20.3 & 101.3 \\
\hline 87 & $808 \mathrm{~B}-25 \mathrm{X}-3,80-81$ & 8.6 & 129.1 & 26.8 & 168.5 & 97.4 & 18.4 & 105.5 \\
\hline 17 & $808 \mathrm{~B}-25 \mathrm{X}-4-\mathrm{A}, 20-21$ & 13.1 & 164.0 & 42.0 & 183.5 & 119.5 & 20.3 & 95.8 \\
\hline 27 & $808 \mathrm{~B}-25 \mathrm{X}-4-\mathrm{B}, 20-2 \mathrm{I}$ & 9.8 & 147.4 & 35.3 & 173.1 & 111.5 & 23.2 & 102.2 \\
\hline 26 & 808B-25X-4-B, $89-90$ & 12.3 & 141.2 & 33.8 & 171.3 & 104.5 & 21.5 & 87.6 \\
\hline 136 & $808 \mathrm{~B}-25 \mathrm{X}-4-\mathrm{A}, 89-90$ & 14.7 & 144.3 & 30.7 & 167.4 & 104.2 & 21.8 & 77.3 \\
\hline 121 & $808 \mathrm{C}-6 \mathrm{R}-2,98-99$ & 14.4 & 153.4 & 24.6 & 145.2 & 126.1 & 19.3 & 98.7 \\
\hline 174 & $808 \mathrm{C}-6 \mathrm{R}-3,54-55$ & 9.1 & 166.8 & 34.3 & 169.7 & 104.8 & 23.8 & 90.3 \\
\hline 14 & $808 \mathrm{C}-6 \mathrm{R}-3-$ A. $76-77$ & 14.0 & 157.8 & 28.9 & 180.7 & 106.1 & 19.7 & 95.6 \\
\hline 42 & $808 \mathrm{C}-6 \mathrm{R}-3-\mathrm{B}, 76-77$ & 8.8 & 151.2 & 24.4 & 183.7 & 107.8 & 14.9 & 92.9 \\
\hline 152 & $808 \mathrm{C}-7 \mathrm{R}-1,33-34$ & 10.0 & 156.3 & 30.1 & 148.5 & 107.4 & 20.5 & 85.4 \\
\hline 88 & $808 C-8 R-1,51-53$ & 12.5 & 140.0 & 32.5 & 165.3 & 96.8 & 18.9 & 91.3 \\
\hline 159 & $808 \mathrm{C}-8 \mathrm{R}-\mathrm{I}, 13 \mathrm{I}-133$ & 11.1 & 153.9 & 24.4 & 172.5 & 108.2 & 17.3 & 87.7 \\
\hline 100 & $808 \mathrm{C}-10 \mathrm{R}-2,105-106$ & 11.0 & 151.2 & 25.7 & 159.6 & 109.5 & 20.5 & 82.1 \\
\hline 134 & $808 C-11 R-1,108-109$ & 6.5 & 148.1 & 26.3 & 161.6 & 95.9 & 22.2 & 75.9 \\
\hline 153 & $808 \mathrm{C}-12 \mathrm{R}-3.24-26$ & 12.9 & 196.9 & 40.2 & 164.2 & 147.1 & 22.4 & 89.2 \\
\hline 179 & $808 \mathrm{C}-12 \mathrm{R}-3,140-142$ & 12.2 & 143.8 & 32.6 & 174.5 & 99.4 & 22.0 & 83.6 \\
\hline
\end{tabular}


Table 5 (continued).

\begin{tabular}{|c|c|c|c|c|c|c|c|c|}
\hline Sample & $\begin{array}{l}\text { Hole, core, section, } \\
\text { interval }(\mathrm{cm})\end{array}$ & Depth (mbsf) & $\mathrm{SiO}_{2}$ & $\mathrm{TiO}_{2}$ & $\mathrm{Al}_{2} \mathrm{O}_{3}$ & $\mathrm{Fe}_{2} \mathrm{O}_{3}$ & $\mathrm{MnO}$ & $\mathrm{MgO}$ \\
\hline 161 & $808 \mathrm{C}-12 \mathrm{R}-4,34-36$ & 409.45 & 61.86 & 0.700 & 14.97 & 6.07 & 0.082 & 2.64 \\
\hline 157 & $808 \mathrm{C}-13 \mathrm{R}-2,47-48$ & 416.27 & 59.67 & 0.713 & 15.21 & 6.54 & 0.097 & 2.58 \\
\hline 102 & $808 \mathrm{C}-15 \mathrm{R}-1.73-74$ & 434.43 & 62.48 & 0.700 & 15.18 & 5.98 & 0.067 & 2.43 \\
\hline 97 & $808 \mathrm{C}-15 \mathrm{R}-3,20-21$ & 436.90 & 60.63 & 0.723 & 15.37 & 6.17 & 0.083 & 2.57 \\
\hline 64 & $808 \mathrm{C}-16 \mathrm{R}-2.53-55$ & 445.04 & 60.39 & 0.712 & 15.79 & 6.69 & 0.122 & 2.70 \\
\hline 60 & $808 C-16 R-4,29-31$ & 447.80 & 61.07 & 0.677 & 16.07 & 6.33 & 0.104 & 2.54 \\
\hline 73 & $808 C-16 R-C C, 12-14$ & 452.53 & 60.35 & 0.684 & 15.89 & 6.71 & 0.129 & 2.60 \\
\hline 55 & $808 \mathrm{C}-18 \mathrm{R}-3,65-67$ & 466.06 & 62.94 & 0.557 & 14.22 & 4.62 & 0.049 & 2.01 \\
\hline 202 & $808 \mathrm{C}-18 \mathrm{R}-3,109-111$ & 466.50 & 60.63 & 0.718 & 14.91 & 5.95 & 0.081 & 2.47 \\
\hline 195 & $808 \mathrm{C}-20 \mathrm{R}-4,81-83$ & 487.02 & 62.92 & 0.724 & 15.56 & 5.97 & 0.126 & 2.42 \\
\hline 68 & $808 \mathrm{C}-2$ IR-1. 7-9 & 491.38 & 61.04 & 0.705 & 17.05 & 6.60 & 0.124 & 2.55 \\
\hline 180 & $808 \mathrm{C}-2$ IR $-3,0-2$ & 494.31 & 61.99 & 0.724 & 15.98 & 5.90 & 0.082 & 2.43 \\
\hline 122 & $808 \mathrm{C}-2 \mathrm{IR}-3,13-15$ & 494.44 & 64.04 & 0.697 & 14.64 & 6.92 & 0.059 & 2.10 \\
\hline 67 & $808 \mathrm{C}-22 \mathrm{R}-2.44-46$ & 502.95 & 62.86 & 0.687 & 16.33 & 6.23 & 0.076 & 2.45 \\
\hline 58 & $808 \mathrm{C}-22 \mathrm{R}-4,39-4 \mathrm{I}$ & 505.90 & 59.58 & 0.702 & 13.50 & 6.18 & 0.076 & 2.50 \\
\hline 104 & $808 \mathrm{C}-23 \mathrm{R}-2,85-86$ & 513.05 & 60.13 & 0.730 & 15.21 & 6.13 & 0.165 & 2.48 \\
\hline 47 & $808 \mathrm{C}-25 \mathrm{R}-1,83-84$ & 530.83 & 61.96 & 0.640 & 15.89 & 5.78 & 0.104 & 2.38 \\
\hline 96 & $808 \mathrm{C}-25 \mathrm{R}-3,74-75$ & 533.74 & 60.92 & 0.679 & 15.26 & 6.02 & 0.104 & 2.41 \\
\hline 140 & $808 \mathrm{C}-26 \mathrm{R}-4,53-55$ & 544.74 & 62.10 & 0.703 & 14.94 & 5.55 & 0.091 & 2.38 \\
\hline 107 & $808 \mathrm{C}-27 \mathrm{R}-1.33-34$ & 549.63 & 59.62 & 0.702 & 14.70 & 6.38 & 0.297 & 2.50 \\
\hline 89 & $808 \mathrm{C}-27 \mathrm{R}-1.134-136$ & 550.65 & 62.61 & 0.668 & 14.79 & 6.21 & 0.109 & 2.42 \\
\hline 131 & $808 \mathrm{C}-27 \mathrm{R}-6.9-10$ & 556.89 & 64.23 & 0.718 & 15.59 & 4.32 & 0.068 & 1.63 \\
\hline 169 & $808 \mathrm{C}-27 \mathrm{R}-6,59-60$ & 557.39 & 61.46 & 0.738 & 14.96 & 5.86 & 0.121 & 2.28 \\
\hline 192 & $808 C-29 R-4,42-44$ & 573.53 & 60.80 & 0.707 & 14.98 & 5.54 & 0.126 & 2.27 \\
\hline 160 & $808 \mathrm{C}-30 \mathrm{R}-3,115-116$ & 582.45 & 60.46 & 0.686 & 14.77 & 5.80 & 0.102 & 2.41 \\
\hline 185 & $808 C-3 \mid R-1,96-98$ & 588.57 & 61.17 & 0.668 & 14.93 & 5.78 & 0.125 & 2.29 \\
\hline 57 & $808 \mathrm{C}-3$ IR-2-A. $113-114$ & 590.23 & 61.31 & 0.708 & 16.53 & 5.94 & 0.094 & 2.50 \\
\hline 69 & $808 \mathrm{C}-31 \mathrm{R}-2-\mathrm{B}, 117-118$ & 590.27 & 61.44 & 0.666 & 15.01 & 7.52 & 0.113 & 2.08 \\
\hline 208 & $808 \mathrm{C}-32 \mathrm{R}-2,65-67$ & 599.46 & 62.61 & 0.715 & 15.28 & 6.37 & 0.063 & 2.46 \\
\hline 61 & $808 \mathrm{C}-34 \mathrm{R}-4,139-141$ & 622.16 & 61.54 & 0.661 & 15.90 & 6.40 & 0.415 & 2.47 \\
\hline 207 & $808 C-35 R-3,26-29$ & 629.47 & 59.47 & 0.685 & 15.23 & 5.61 & 0.159 & 2.32 \\
\hline 167 & $808 C-35 R-5.133-135$ & 632.55 & 62.46 & 0.707 & 15.10 & 5.98 & 0.120 & 2.42 \\
\hline 53 & $808 \mathrm{C}-36 \mathrm{R}-4.82-83$ & 641.22 & 61.78 & 0.691 & 16.65 & 6.25 & 0.133 & 2.51 \\
\hline 62 & $808 \mathrm{C}-38 \mathrm{R}-3,146-148$ & 659.37 & 55.16 & 0.637 & 15.29 & 5.50 & 0.255 & 2.31 \\
\hline 126 & $808 \mathrm{C}-39 \mathrm{R}-1,98-100$ & 665.49 & 63.88 & 0.617 & 15.80 & 5.17 & 0.084 & 2.66 \\
\hline 81 & $808 \mathrm{C}-40 \mathrm{R}-1.75-77$ & 674.96 & 62.17 & 0.682 & 16.11 & 5.58 & 0.103 & 2.47 \\
\hline 71 & $808 C-42 R-1,135-137$ & 694.86 & 54.97 & 0.614 & 14.80 & 5.29 & 0.244 & 2.29 \\
\hline 11 & $808 \mathrm{C}-42 \mathrm{R}-3,1-3$ & 696.52 & 56.21 & 0.610 & 14.77 & 5.56 & 0.209 & 2.30 \\
\hline 66 & $808 \mathrm{C}-43 \mathrm{R}-3,20-22$ & 696.71 & 62.95 & 0.694 & 16.81 & 5.89 & 0.076 & 2.59 \\
\hline 188 & $808 \mathrm{C}-44 \mathrm{R}-2,46-47$ & 714.36 & 58.46 & 0.627 & 15.43 & 5.84 & 0.161 & 2.25 \\
\hline 163 & $808 C-45 R-3,52-54$ & 725.53 & 60.72 & 0.434 & 16.31 & 7.37 & 0.040 & 3.47 \\
\hline 130 & $808 C-45 R-3.73-75$ & 725.74 & 61.62 & 0.671 & 15.19 & 5.60 & 0.104 & 2.35 \\
\hline 156 & $808 \mathrm{C}-45 \mathrm{R}-4,50-52$ & 727.01 & 60.21 & 0.669 & 14.89 & 6.07 & 0.109 & 2.38 \\
\hline 54 & $808 \mathrm{C}-46 \mathrm{R}-1,40-42$ & 732.11 & 61.57 & 0.670 & 16.75 & 5.98 & 0.075 & 2.40 \\
\hline 103 & $808 \mathrm{C}-47 \mathrm{R}-4,26-28$ & 746.17 & 61.03 & 0.482 & 15.27 & 6.15 & 0.045 & 2.32 \\
\hline 203 & $808 \mathrm{C}-47 \mathrm{R}-4,49-51$ & 746.40 & 62.22 & 0.654 & 15.16 & 5.42 & 0.051 & 2.24 \\
\hline 189 & 808C-48R-5, 88-89 & 757.98 & 58.47 & 0.569 & 13.04 & 4.65 & 0.123 & 2.01 \\
\hline 56 & $808 C-49 R-3,75-77$ & 764.46 & 61.21 & 0.648 & 15.82 & 5.79 & 0.066 & 2.27 \\
\hline 110 & $808 \mathrm{C}-50 \mathrm{R}-3.58-60$ & 773.99 & 59.16 & 0.649 & 15.30 & 6.47 & 0.037 & 2.57 \\
\hline 5 & $808 \mathrm{C}-51 \mathrm{R}-2,12-14$ & 781.73 & 50.87 & 0.558 & 13.45 & 5.32 & 0.381 & 2.03 \\
\hline 25 & $808 C-52 R-2,7-9$ & 791.38 & 58.27 & 0.603 & 15.36 & 6.60 & 0.056 & 2.37 \\
\hline 77 & $808 C-53 R-5.127-129$ & 803.68 & 57.77 & 0.591 & 14.44 & 5.14 & 0.118 & 1.98 \\
\hline 72 & $808 \mathrm{C}-54 \mathrm{R}-2,87-91$ & 811.48 & 65.29 & 0.616 & 15.26 & 4.57 & 0.057 & 1.97 \\
\hline 12 & $808 \mathrm{C}-54 \mathrm{R}-2,100-102$ & 811.61 & 61.59 & 0.594 & 16.11 & 5.35 & 0.057 & 2.34 \\
\hline 176 & $808 C-55 R-3,70-72$ & 820.96 & 61.33 & 0.710 & 15.52 & 6.17 & 0.046 & 2.54 \\
\hline 201 & $808 \mathrm{C}-56 \mathrm{R}-3,37-39$ & 831.81 & 62.31 & 0.705 & 15.73 & 6.08 & 0.045 & 2.44 \\
\hline 63 & $808 \mathrm{C}-57 \mathrm{R}-1.50-52$ & 838.61 & 62.42 & 0.715 & 17.25 & 6.32 & 0.067 & 2.51 \\
\hline 194 & $808 \mathrm{C}-58 \mathrm{R}-2.116-118$ & 859.77 & 60.53 & 0.676 & 15.36 & 5.52 & 0.070 & 2.32 \\
\hline 98 & $808 \mathrm{C}-59 \mathrm{R}-4,29-30$ & 861.89 & 60.12 & 0.621 & 16.51 & 5.73 & 0.058 & 2.43 \\
\hline 151 & $808 \mathrm{C}-60 \mathrm{R}-3,69-70$ & 870.49 & 61.89 & 0.682 & 15.56 & 5.73 & 0.085 & 2.39 \\
\hline 46 & $808 C-6 \mid R-3.94-95$ & 880.44 & 62.29 & 0.693 & 16.53 & 6.12 & 0.085 & 2.47 \\
\hline 210 & $808 \mathrm{C}-61 \mathrm{R}-4,72-73$ & 881.64 & 59.77 & 0.878 & 17.05 & 7.61 & 0.054 & 2.78 \\
\hline 70 & $808 \mathrm{C}-62 \mathrm{R}-1,114-116$ & 887.55 & 62.91 & 0.734 & 17.49 & 5.93 & 0.086 & 2.35 \\
\hline 65 & $808 \mathrm{C}-63 \mathrm{R}-3,16-18$ & 898.95 & 62.73 & 0.645 & 17.06 & 5.74 & 0.095 & 2.54 \\
\hline 48 & $808 \mathrm{C}-64 \mathrm{R}-4,58-60$ & 910.59 & 63.53 & 0.759 & 18.66 & 6.43 & 0.136 & 2.54 \\
\hline 196 & $808 C-65 R-1,98-100$ & 916.09 & 61.50 & 0.702 & 15.95 & 6.03 & 0.072 & 2.33 \\
\hline 49 & $808 \mathrm{C}-66 \mathrm{R}-2,94-95$ & 927.24 & 62.57 & 0.727 & 18.37 & 6.55 & 0.107 & 2.48 \\
\hline 177 & $808 \mathrm{C}-67 \mathrm{R}-1.52-54$ & 935.03 & 42.23 & 0.447 & 11.04 & 4.12 & 1.416 & 1.79 \\
\hline 191 & $808 \mathrm{C}-68 \mathrm{R}-2,14-16$ & 945.85 & 60.55 & 0.697 & 15.57 & 5.80 & 0.151 & 2.43 \\
\hline 3 & $808 \mathrm{C}-69 \mathrm{R}-3,26-28$ & 957.07 & 62.34 & 0.725 & 17.51 & 6.26 & 0.124 & 2.66 \\
\hline 2 & $808 \mathrm{C}-69 \mathrm{R}-3,120-122$ & 958.01 & 62.51 & 0.706 & 17.28 & 6.26 & 0.086 & 2.59 \\
\hline 94 & $808 \mathrm{C}-69 \mathrm{R}-5,36-38$ & 960.17 & 60.74 & 0.701 & 16.01 & 6.06 & 0.081 & 2.55 \\
\hline 4 & $808 \mathrm{C}-71 \mathrm{R}-2,106-107$ & 964.48 & 57.68 & 0.590 & 17.26 & 5.68 & 0.138 & 2.53 \\
\hline 186 & $808 \mathrm{C}-71 \mathrm{R}-4,87-88$ & 978.47 & 60.68 & 0.681 & 15.85 & 7.09 & 0.196 & 2.43 \\
\hline 10 & $808 \mathrm{C}-72 \mathrm{R}-2,12-14$ & 984.43 & 59.73 & 0.665 & 16.31 & 6.30 & 0.084 & 2.33 \\
\hline 138 & $808 \mathrm{C}-73 \mathrm{R}-2,74-76$ & 994.35 & 65.18 & 0.717 & 16.12 & 6.29 & 0.081 & 2.53 \\
\hline 158 & $808 \mathrm{C}-74 \mathrm{R}-2,35-36$ & 1003.65 & 63.46 & 0.706 & 16.18 & 6.19 & 0.084 & 2.40 \\
\hline 193 & $808 C-75 R-1,60-61$ & 1011,90 & 62.50 & 0.667 & 16.74 & 6.30 & 0.119 & 2.53 \\
\hline 206 & $808 \mathrm{C}-76 \mathrm{R}-1,0-2$ & 1020.46 & 62.58 & 0.656 & 16.19 & 6.40 & 0.078 & 2.32 \\
\hline 45 & $808 \mathrm{C}-77 \mathrm{R}-1,38-40$ & 1030.33 & 63.22 & 0.698 & 17.51 & 6.49 & 0.096 & 2.47 \\
\hline 30 & 808C-79R-1-A. $37-39$ & 1048.68 & 62.40 & 0.687 & 17.72 & 6.63 & 0.115 & 2.44 \\
\hline 40 & $808 \mathrm{C}-79 \mathrm{R}-1-\mathrm{B}, 37-39$ & 1048.68 & 63.01 & 0.697 & 17.52 & 6.64 & 0.115 & 2.44 \\
\hline 183 & $808 \mathrm{C}-80 \mathrm{R}-1,60-62$ & 1058.41 & 60.24 & 0.677 & 15.72 & 5.84 & 0.339 & 2.44 \\
\hline 127 & $808 \mathrm{C}-81 \mathrm{R}-1.115-117$ & 1068.26 & 63.73 & 0.706 & 16.31 & 6.22 & 0.111 & 2.63 \\
\hline 199 & $808 \mathrm{C}-82 \mathrm{R}-1,34-35$ & 1076.84 & 63.59 & 0.708 & 16.15 & 6.21 & 0.079 & 2.54 \\
\hline 95 & $808 C-82 R-2,56-57$ & 1078.52 & 57.30 & 0.655 & 14.93 & 6.33 & 3.349 & 2.42 \\
\hline 181 & $808 \mathrm{C}-83 \mathrm{R}-1,35-37$ & 1086.16 & 63.14 & 0.706 & 16.21 & 6.29 & 0.185 & 2.47 \\
\hline 205 & $808 C-83 R-2,57-58$ & 1087.87 & 27.74 & 0.266 & 7.562 & 6.21 & 14.696 & 1.78 \\
\hline 106 & $808 \mathrm{C}-84 \mathrm{R}-1,55-57$ & 1092.56 & 62.02 & 0.722 & 16.03 & 6.36 & 0.459 & 2.44 \\
\hline
\end{tabular}


Table 5 (continued).

\begin{tabular}{|c|c|c|c|c|c|c|c|c|}
\hline Sample & $\begin{array}{l}\text { Hole, core, section, } \\
\text { interval }(\mathrm{cm})\end{array}$ & $\mathrm{Nb}$ & $\mathrm{Zr}$ & $\mathbf{Y}$ & Sr & $\mathrm{Rb}$ & Ga & $\mathrm{Zn}$ \\
\hline 161 & $808 \mathrm{C}-12 \mathrm{R}-4,34-36$ & 11.9 & 146.1 & 32.7 & 238.0 & 66.6 & 17.5 & 65.9 \\
\hline 157 & $808 \mathrm{C}-13 \mathrm{R}-2,47-48$ & 9.6 & 156.5 & 34.3 & 149.4 & 125.0 & 20.7 & 85.7 \\
\hline 102 & $808 \mathrm{C}-15 \mathrm{R}-1,73-74$ & 11.7 & 158.2 & 26.2 & 119.9 & 118.1 & 18.2 & 91.0 \\
\hline 97 & $808 C-15 R-3,20-21$ & 12.9 & 150.3 & 25.5 & 148.5 & 109.2 & 17.8 & 100.7 \\
\hline 64 & $808 \mathrm{C}-16 \mathrm{R}-2,53-55$ & 12.9 & 168.4 & 30.3 & 153.4 & 115.1 & 16.8 & 77.7 \\
\hline 60 & $808 \mathrm{C}-16 \mathrm{R}-4,29-31$ & 13.5 & 157.8 & 30.2 & 158.6 & 118.7 & 18.3 & 87.0 \\
\hline 73 & 808C-16R-CC, $12-14$ & 9.7 & 188.4 & 34.3 & 145.9 & 132.9 & 22.5 & 89.7 \\
\hline 55 & $808 C-18 R-3,65-67$ & 7.2 & 76.8 & 13.0 & 76.9 & 50.0 & 10.6 & 35.1 \\
\hline 202 & 808C-18R-3, 109-111 & 10.8 & 160.5 & 32.1 & 179.2 & 102.6 & 17.2 & 80.5 \\
\hline 195 & $808 \mathrm{C}-20 \mathrm{R}-4,81-83$ & 16.9 & 179.4 & 31.6 & 152.7 & 138.5 & 19.6 & 86.0 \\
\hline 68 & $808 \mathrm{C}-2$ IR-1. 7-9 & 14.0 & 135.5 & 29.5 & 193.2 & 110.2 & 18.0 & 83.2 \\
\hline 180 & $808 \mathrm{C}-2 \mathrm{IR}-3,0-2$ & 8.3 & 161.1 & 32.1 & 142.0 & 113.7 & 18.2 & 79.6 \\
\hline 122 & $808 \mathrm{C}-21 \mathrm{R}-3,13-15$ & 14.0 & 207.0 & 27.9 & 202.4 & 110.4 & 19.0 & 78.0 \\
\hline 67 & $808 \mathrm{C}-22 \mathrm{R}-2,44-46$ & 5.2 & 144.9 & 25.7 & 147.6 & 108.4 & 14.5 & 81.2 \\
\hline 58 & $808 C-22 R-4,39-41$ & 5.3 & 75.1 & 13.1 & 76.0 & 55.4 & 12.9 & 48.7 \\
\hline 104 & $808 \mathrm{C}-23 \mathrm{R}-2,85-86$ & 10.8 & 177.1 & 30.2 & 126.3 & 121.5 & 20.1 & 81.0 \\
\hline 47 & $808 \mathrm{C}-25 \mathrm{R}-1,83-84$ & 16.1 & 154.1 & 26.1 & 148.3 & 119.0 & 18.4 & 85.8 \\
\hline 96 & $808 C-25 R-3,74-75$ & 12.9 & 157.3 & 30.1 & 135.0 & 124.3 & 21.1 & 86.8 \\
\hline 140 & $808 C-26 R-4,53-55$ & 11.5 & 164.3 & 36.8 & 166.1 & 108.8 & 19.4 & 86.6 \\
\hline 107 & $808 C-27 R-1,33-34$ & 18.2 & 179.2 & 34.5 & 175.4 & 136.5 & 20.6 & 78.5 \\
\hline 89 & $808 \mathrm{C}-27 \mathrm{R}-1,134-136$ & 10.9 & 140.1 & 26.6 & 138.6 & 109.1 & 21.4 & 95.1 \\
\hline 131 & $808 C-27 R-6,9-10$ & 14.9 & 296.4 & 42.1 & 192.2 & 112.4 & 21.2 & 57.0 \\
\hline 169 & $808 \mathrm{C}-27 \mathrm{R}-6,59-60$ & 17.2 & 187.6 & 32.2 & 144.9 & 129.0 & 20.6 & 83.6 \\
\hline 192 & $808 \mathrm{C}-29 \mathrm{R}-4,42-44$ & 14.1 & 145.8 & 33.0 & 151.5 & 102.7 & 21.1 & 87.8 \\
\hline 160 & $808 C-30 R-3,115-116$ & 16.4 & 159.4 & 29.5 & 182.5 & 110.3 & 20.1 & 83.0 \\
\hline 185 & 808C-3IR-1, 96-98 & 10.9 & 162.7 & 28.8 & 179.8 & 122.5 & 15.0 & 76.8 \\
\hline 57 & 808C-31R-2-A, $113-114$ & 7.0 & 147.5 & 23.8 & 161.1 & 123.4 & 15.3 & 96.7 \\
\hline 69 & 808C-3IR-2-B. $117-118$ & 3.8 & 184.9 & 33.2 & 178.9 & 106.5 & 17.1 & 91.2 \\
\hline 208 & $808 \mathrm{C}-32 \mathrm{R}-2,65-67$ & 16.8 & 168.5 & 36.3 & 145.8 & 109.0 & 20.0 & 100.4 \\
\hline 61 & $808 \mathrm{C}-34 \mathrm{R}-4,139-141$ & 10.0 & 142.9 & 29.0 & 147.0 & 101.4 & 15.6 & 90.0 \\
\hline 207 & $808 C-35 R-3,26-29$ & 12.4 & 146.8 & 32.9 & 184.1 & 114.2 & 15.9 & 79.0 \\
\hline 167 & $808 C-35 R-5,133-135$ & 14.4 & 153.3 & 29.7 & 162.8 & 109.5 & 19.3 & 94.6 \\
\hline 53 & $808 \mathrm{C}-36 \mathrm{R}-4,82-83$ & 14.8 & 152.0 & 29.2 & 137.8 & 134.6 & 16.1 & 94.2 \\
\hline 62 & $808 C-38 R-3,146-148$ & 10.7 & 134.9 & 36.3 & 256.3 & 118.5 & 17.0 & 89.2 \\
\hline 126 & $808 \mathrm{C}-39 \mathrm{R}-1,98-100$ & 10.7 & 168.0 & 29.8 & 176.2 & 114.1 & 25.0 & 92.9 \\
\hline 81 & $808 \mathrm{C}-40 \mathrm{R}-1.75-77$ & 11.6 & 162.1 & 32.6 & 159.0 & 137.5 & 22.9 & 109.5 \\
\hline 71 & $808 \mathrm{C}-42 \mathrm{R}-1,135-137$ & 10.8 & 142.5 & 24.7 & 287.4 & 124.8 & 21.8 & 85.2 \\
\hline 11 & $808 \mathrm{C}-42 \mathrm{R}-3,1-3$ & 16.2 & 131.1 & 26.5 & 261.7 & 124.7 & 20.8 & 84.2 \\
\hline 66 & $808 C-43 R-3,20-22$ & 14.5 & 182.7 & 29.8 & 137.0 & 145.8 & 17.7 & 104.7 \\
\hline 188 & $808 \mathrm{C}-44 \mathrm{R}-2,46-47$ & 11.7 & 159.5 & 37.0 & 213.2 & 121.6 & 20.0 & 95.1 \\
\hline 163 & $808 \mathrm{C}-45 \mathrm{R}-3,52-54$ & 9.4 & 248.3 & 35.8 & 168.4 & 7.0 & 17.6 & 45.8 \\
\hline 130 & $808 \mathrm{C}-45 \mathrm{R}-3,73-75$ & 14.5 & 151.7 & 32.4 & 187.9 & 123.9 & 19.5 & 95.8 \\
\hline 156 & $808 C-45 R-4,50-52$ & 13.4 & 152.1 & 30.9 & 178.3 & 123.5 & 18.1 & 95.5 \\
\hline 54 & $808 \mathrm{C}-46 \mathrm{R}-1,40-42$ & 10.3 & 124.7 & 26.2 & 182.6 & 105.8 & 18.7 & 101.2 \\
\hline 103 & $808 C-47 R-4,26-28$ & 13.0 & 133.8 & 36.9 & 208.6 & 70.7 & 18.4 & 73.7 \\
\hline 203 & $808 C-47 R-4,49-51$ & 13.3 & 138.2 & 26.4 & 171.4 & 122.2 & 19.0 & 101.9 \\
\hline 189 & $808 C-48 R-5,88-89$ & 15.9 & 123.9 & 37.4 & 262.8 & 116.0 & 14.7 & 73.9 \\
\hline 56 & $808 \mathrm{C}-49 \mathrm{R}-3,75-77$ & 9.7 & 136.6 & 30.0 & 170.6 & 118.1 & 14.8 & 98.4 \\
\hline 110 & $808 \mathrm{C}-50 \mathrm{R}-3,58-60$ & 9.0 & 166.3 & 31.8 & 166.5 & 101.3 & 17.7 & 73.4 \\
\hline 5 & $808 \mathrm{C}-51 \mathrm{R}-2,12-14$ & 9.1 & 134.5 & 29.2 & 245.8 & 116.7 & 17.5 & 95.7 \\
\hline 25 & $808 C-52 R-2,7-9$ & 11.5 & 160.8 & 37.4 & 208.9 & 114.2 & 15.9 & 99.0 \\
\hline 77 & $808 C-53 R-5,127-129$ & 9.3 & 128.9 & 30.4 & 237.0 & 105.8 & 16.5 & 88.7 \\
\hline 72 & $808 C-54 R-2,87-91$ & 8.8 & 143.7 & 25.5 & 211.8 & 114.8 & 14.4 & 88.3 \\
\hline 12 & $808 C-54 R-2,100-102$ & 11.4 & 134.9 & 23.5 & 219.2 & 99.5 & 18.9 & 88.7 \\
\hline 176 & $808 \mathrm{C}-55 \mathrm{R}-3,70-72$ & 11.0 & 172.6 & 25.4 & 163.4 & 114.4 & 23.8 & 92.9 \\
\hline 201 & $808 C-56 R-3,37-39$ & 10.6 & 165.6 & 29.3 & 137.9 & 116.1 & 19.4 & 102.4 \\
\hline 63 & $808 \mathrm{C}-57 \mathrm{R}-1.50-52$ & 16.8 & 158.1 & 36.6 & 114.1 & 128.2 & 17.7 & 105.1 \\
\hline 194 & $808 C-58 R-2,116-118$ & 12.2 & 171.8 & 37.9 & 200.0 & 123.7 & 22.2 & 92.3 \\
\hline 98 & $808 C-59 R-4,29-30$ & 12.3 & 146.7 & 35.5 & 164.3 & 99.5 & 18.3 & 90.5 \\
\hline 151 & $808 \mathrm{C}-60 \mathrm{R}-3,69-70$ & 11.7 & 176.3 & 37.7 & 183.9 & 147.6 & 19.7 & 95.8 \\
\hline 46 & $808 C-6 \mid R-3.94-95$ & 10.7 & 162.9 & 34.3 & 140.0 & 129.6 & 20.7 & 113.5 \\
\hline 210 & $808 \mathrm{C}-61 \mathrm{R}-4,72-73$ & 8.9 & 175.4 & 20.0 & 133.9 & 76.9 & 15.4 & 90.4 \\
\hline 70 & $808 \mathrm{C}-62 \mathrm{R}-1,114-116$ & 11.2 & 155.1 & 37.4 & 135.7 & 127.8 & 17.4 & 108.6 \\
\hline 65 & $808 C-63 R-3,16-18$ & 12.7 & 142.4 & 30.7 & 123.4 & 113.6 & 17.7 & 102.2 \\
\hline 48 & $808 \mathrm{C}-64 \mathrm{R}-4,58-60$ & 13.6 & 170.8 & 37.0 & 102.2 & 159.6 & 22.0 & 103.1 \\
\hline 196 & $808 C-65 R-1,98-100$ & 10.8 & 157.4 & 28.5 & 139.4 & 129.3 & 23.9 & 83.0 \\
\hline 49 & $808 \mathrm{C}-66 \mathrm{R}-2,94-95$ & 16.0 & 173.9 & 35.3 & 114.6 & 167.9 & 22.7 & 109.9 \\
\hline 177 & $808 C-67 R-1.52-54$ & 14.5 & 119.3 & 36.4 & 225.8 & 88.7 & 11.8 & 72.1 \\
\hline 191 & $808 \mathrm{C}-68 \mathrm{R}-2,14-16$ & 14.6 & 158.2 & 34.4 & 172.4 & 132.6 & 19.8 & 98.9 \\
\hline 3 & $808 \mathrm{C}-69 \mathrm{R}-3,26-28$ & 14.4 & 165.0 & 32.0 & 116.6 & 157.4 & 24.8 & 105.3 \\
\hline 2 & $808 \mathrm{C}-69 \mathrm{R}-3,120-122$ & 14.0 & 167.4 & 33.7 & 120.2 & 152.7 & 21.1 & 107.8 \\
\hline 94 & $808 \mathrm{C}-69 \mathrm{R}-5,36-38$ & 15.8 & 163.1 & 37.8 & 129.9 & 147.8 & 18.2 & 124.3 \\
\hline 4 & $808 \mathrm{C}-7$ IR-2, 106-107 & 15.1 & 144.6 & 27.8 & 217.4 & 125.4 & 20,8 & 106.8 \\
\hline 186 & $808 \mathrm{C}-71 \mathrm{R}-4,87-88$ & 10.2 & 166.1 & 37.9 & 171.0 & 124.9 & 14.3 & 83.8 \\
\hline 10 & $808 C-72 R-2,12-14$ & 13.6 & 138.9 & 25.8 & 127.4 & 130.3 & 20.1 & 114.0 \\
\hline 138 & $808 \mathrm{C}-73 \mathrm{R}-2,74-76$ & 13.6 & 171.7 & 30.7 & 105.0 & 161.9 & 26.8 & 108.2 \\
\hline 158 & $808 \mathrm{C}-74 \mathrm{R}-2,35-36$ & 15.4 & 176.7 & 41.2 & 105.7 & 150.4 & 22.5 & 111.2 \\
\hline 193 & $808 \mathrm{C}-75 \mathrm{R}-1,60-61$ & 14.9 & 151.0 & 29.9 & 108.5 & 122.9 & 21.4 & 95.1 \\
\hline 206 & $808 \mathrm{C}-76 \mathrm{R}-1,0-2$ & 8.7 & 155.2 & 32.6 & 91.7 & 142.6 & 24.0 & 102.4 \\
\hline 45 & $808 \mathrm{C}-77 \mathrm{R}-1,38-40$ & 11.2 & 145.7 & 31.0 & 90.8 & 144.7 & 20.7 & 95.3 \\
\hline 30 & 808 C-79R-1-A, $37-39$ & 15.7 & 164,4 & 32.0 & 101.4 & 167.7 & 21.9 & 107.1 \\
\hline 40 & $808 \mathrm{C}-79 \mathrm{R}-1-\mathrm{B}, 37-39$ & 14.9 & 141.4 & 20.4 & 92.2 & 142.6 & 24.1 & 108.1 \\
\hline 183 & $808 C-80 R-1,60-62$ & 14.9 & 145.7 & 26.3 & 154.0 & 133.9 & 16.0 & 93.2 \\
\hline 127 & $808 \mathrm{C}-81 \mathrm{R}-1.115-117$ & 11.3 & 162.8 & 29.9 & 112.8 & 169.9 & 24.8 & 99.3 \\
\hline 199 & $808 \mathrm{C}-82 \mathrm{R}-1.34-35$ & 19.7 & 158.2 & 27.8 & 94.8 & 146.8 & 21.7 & 98.6 \\
\hline 95 & $808 \mathrm{C}-82 \mathrm{R}-2,56-57$ & 7.9 & 147.7 & 33.9 & 115.7 & 159.2 & 24.0 & 95.8 \\
\hline 181 & $808 C-83 R-1,35-37$ & 15.3 & 157.2 & 28.5 & 95.3 & 156.8 & 17.9 & 96.8 \\
\hline 205 & $808 C-83 R-2,57-58$ & 14.1 & 73.6 & 425.2 & 556.1 & 90.3 & 8.4 & 59.2 \\
\hline 106 & $808 \mathrm{C}-84 \mathrm{R}-1.55-57$ & 16.6 & 139.4 & 34.2 & 97.3 & 140.5 & 18.4 & 93.4 \\
\hline
\end{tabular}


Table 5 (continued).

\begin{tabular}{|c|c|c|c|c|c|c|c|c|c|c|c|c|c|c|}
\hline Sample & $\begin{array}{l}\text { Hole, core, section. } \\
\text { interval }(\mathrm{cm})\end{array}$ & Depth (mbsf) & $\mathrm{SiO}_{2}$ & $\mathrm{TiO}_{2}$ & $\mathrm{Al}_{2} \mathrm{O}_{3}$ & $\mathrm{Fe}_{2} \mathrm{O}_{3}$ & $\mathrm{MnO}$ & $\mathrm{MgO}$ & $\mathrm{CaO}$ & $\mathrm{Na}_{2} \mathrm{O}$ & $\mathrm{K}_{2} \mathrm{O}$ & $\mathrm{P}_{2} \mathrm{O}_{5}$ & LOI & Total \\
\hline 150 & $808 C-85 R-1.59-61$ & 1098.90 & 20.75 & 0.241 & 5.767 & 5.76 & 11.939 & 1.46 & 22.4 & 1.27 & 1.15 & 15.818 & 13.87 & 100.500 \\
\hline 139 & $808 \mathrm{C}-85 \mathrm{R}-2,68-70$ & 1099.59 & 62.74 & 0.715 & 16.26 & 6.61 & 0.083 & 2.44 & 0.334 & 1.40 & 3.47 & 0.073 & 4.59 & 99.090 \\
\hline 211 & $808 \mathrm{C}-86 \mathrm{R}-2,110-112$ & 1110.61 & 25.32 & 0.257 & 6.908 & 4.59 & 10.500 & 1.80 & 37.6 & 0.954 & 1.04 & 6.212 & 4.73 & 100.150 \\
\hline 51 & $808 \mathrm{C}-87 \mathrm{R}-1.78-79$ & 1117.98 & 62.70 & 0.694 & 18.05 & 6.11 & 0.076 & 2.53 & 0.456 & 1.36 & 3.46 & 0.067 & 5.04 & 100.960 \\
\hline 190 & $808 \mathrm{C}-88 \mathrm{R}-2.21-24$ & 1128.62 & 42.05 & 0.437 & 11.20 & 4.82 & 2.196 & 1.84 & 15.6 & 1.08 & 2.22 & 0.781 & 15.57 & 98.070 \\
\hline 197 & $808 \mathrm{C}-89 \mathrm{R}-1,58-60$ & 1137.16 & 41.03 & 0.320 & 12.81 & 5.72 & 2.375 & 2.05 & 14.9 & 1.42 & 1.50 & 0.141 & 15,18 & 96.090 \\
\hline 155 & $808 \mathrm{C}-89 \mathrm{R}-1.126-128$ & 1137.84 & 56.89 & 0.636 & 14.90 & 5.77 & 0.249 & 2.22 & 5.00 & 1.28 & 3.01 & 0.069 & 7.71 & 98.060 \\
\hline 154 & $808 \mathrm{C}-90 \mathrm{R}-1.98-100$ & 1147.29 & 60.40 & 0.707 & 16.11 & 6.40 & 0.193 & 2.46 & 2,41 & 1.36 & 3.16 & 0.072 & 5.75 & 99.330 \\
\hline 141 & $808 \mathrm{C}-92 \mathrm{R}-1,110-113$ & 1166.73 & 60.84 & 0.699 & 15.82 & 6.28 & 0.190 & 2.36 & 2.10 & 1.35 & 3.19 & 0.072 & 5.45 & 98.680 \\
\hline 105 & 808 C-92R-CC. $8-10$ & 1167.72 & 41.58 & 0.432 & 10.78 & 4.70 & 2.145 & 1.76 & 16.6 & 0.984 & 2.17 & 0.369 & 16.58 & 98.360 \\
\hline 182 & $808 C-93 R-1,76-78$ & 1176.07 & 62.07 & 0.704 & 16.05 & 6.63 & 0.191 & 2.41 & 0.952 & 1.33 & 3.21 & 0.082 & 4.76 & 98.720 \\
\hline 50 & $808 \mathrm{C}-94 \mathrm{R}-1.92-94$ & 1185.83 & 62.94 & 0.712 & 16.38 & 6.00 & 0.122 & 2.27 & 0.655 & 1.24 & 3.43 & 0.073 & 5.09 & 99.360 \\
\hline 187 & $808 \mathrm{C}-94 \mathrm{R}-1,117-119$ & 1186.11 & 62.01 & 0.722 & 16.32 & 6.60 & 0.154 & 2.33 & 0.865 & 1.27 & 3.43 & 0.068 & 4.89 & 99.010 \\
\hline 175 & $808 C-95 R-1,22-24$ & 1194.80 & 61.54 & 0.732 & 16.53 & 6.26 & 0.122 & 2.27 & 1.04 & 1.26 & 3.45 & 0.068 & 4.87 & 98.450 \\
\hline 79 & $808 C-95 R-1,131-133$ & 1195.89 & 37.62 & 0.424 & 10.70 & 4.40 & 1.506 & 1.52 & 19.6 & 0.853 & 2.25 & 2.754 & 16.53 & 98.370 \\
\hline 178 & $808 \mathrm{C}-96 \mathrm{R}-1.40-42$ & 1204.99 & 61.74 & 0.701 & 16.32 & 6.71 & 0.138 & 2.25 & 0.715 & 1.13 & 3.56 & 0.081 & 4.60 & 98.310 \\
\hline 52 & $808 \mathrm{C}-97 \mathrm{R}-1.45-46$ & 1214,45 & 60.27 & 0.687 & 17.45 & 6.51 & 0.292 & 2.41 & 1.85 & 1.27 & 3.63 & 0.075 & 5.97 & 100.730 \\
\hline 129 & 808C-98R-1-A. $91-92$ & 1224.61 & 61.34 & 0.736 & 16.45 & 6.85 & 0.232 & 2.34 & 1.78 & 1.20 & 3.82 & 0.742 & 4.49 & 100.290 \\
\hline 133 & 808C-98R-1-B, 91-92 siderite? & 1224.61 & 62.55 & 0.738 & 16.48 & 8.12 & 0.176 & 2.32 & 0.470 & 1.12 & 4.08 & 0.084 & 4.14 & 100.600 \\
\hline 164 & $808 \mathrm{C}-99 \mathrm{R}-1.86-88$ & 1234.27 & 61.76 & 0.698 & 16.02 & 7.32 & 2.210 & 2.57 & 0.517 & 1.23 & 3.63 & 0.079 & 4.35 & 100.650 \\
\hline 198 & $808 \mathrm{C}-100 \mathrm{R}-1,39-41$ & 1243.40 & 65.51 & 0.696 & 14.37 & 5.77 & 0.151 & 2.24 & 1.14 & 1.65 & 3.26 & 0.086 & 3.31 & 98.690 \\
\hline 44 & $808 \mathrm{C}-10 \mid \mathrm{R}-1.85-87$ & 1253.06 & 75.65 & 0.222 & 11.93 & 1.59 & 0.058 & 0.608 & 0.646 & 2.93 & 2.21 & 0.154 & 3.58 & 100.960 \\
\hline 200 & $808 C-101 R-3,55-57$ & 1255.74 & 73.92 & 0.230 & 11.72 & 1.74 & 0.063 & 0.634 & 0.643 & 2.89 & 1.21 & 0.144 & 3.96 & 99.460 \\
\hline 9 & $808 C-101 R-4,62-64$ & 1257.31 & 72.93 & 0.405 & 10.86 & 4.85 & 0.135 & 1.41 & 1.10 & 1.40 & 3.09 & 0.058 & 3.67 & 100.260 \\
\hline 135 & $808 \mathrm{C}-102 \mathrm{R}-2,80-82$ & 1263.81 & 66.84 & 0.345 & 7.495 & 4.68 & 0.370 & 1.42 & 6.31 & 0.842 & 3.11 & 0.118 & 6.79 & 98.560 \\
\hline 209 & $808 \mathrm{C}-102 \mathrm{R}-3,133-134$ & 1265.83 & 65.29 & 0.160 & 15.88 & 1.93 & 0.138 & 1.68 & 2.61 & 1.46 & 1.82 & 0.050 & 5.85 & 98.430 \\
\hline 173 & $808 \mathrm{C}-103 \mathrm{R}-1,121-123$ & 1272.14 & 65.46 & 0.563 & 13.12 & 7.31 & 0.163 & 1.95 & 0.456 & 1.05 & 5.66 & 0.103 & 2.94 & 99.030 \\
\hline III & $808 C-103 R-3.16-18$ & 1274.07 & 61.88 & 0.712 & 15.01 & 5.89 & 0.080 & 2.45 & 2.44 & 2.21 & 2.74 & 0.122 & 5.03 & 99.090 \\
\hline 1 & $808 C-103 R-3,40-42$ & 1274.31 & 70.47 & 0.406 & 14.46 & 1.12 & 0.047 & 0.897 & 2.62 & 0.924 & 0.897 & 0.047 & 5.64 & 100.280 \\
\hline 109 & $808 \mathrm{C}-104 \mathrm{R}-1,74-75$ & 1281.15 & 54.88 & 0.468 & 11.68 & 5.37 & 0.323 & 2.15 & 9.62 & 1.01 & 4.25 & 0.087 & 10.07 & 100.210 \\
\hline 15 & $808 \mathrm{C}-106 \mathrm{R}-2,103-108$ & 1301.54 & 49.08 & 0.473 & 12.17 & 7.01 & 0.062 & 2.99 & 10.7 & 0.650 & 6.04 & 0.098 & 10.76 & 100.250 \\
\hline 39 & $808 \mathrm{C}-106 \mathrm{R}-2,103-108$ & 1301.54 & 49.13 & 0.472 & 11.83 & 7.04 & 0.062 & 2.97 & 10.9 & 0.705 & 6.07 & 0.098 & 10.97 & 100.320 \\
\hline
\end{tabular}


Table 5 (continued).

\begin{tabular}{|c|c|c|c|c|c|c|c|c|c|c|c|c|c|c|c|c|c|c|c|}
\hline Sample & $\begin{array}{l}\text { Hole, core, section. } \\
\text { interval }(\mathrm{cm})\end{array}$ & $\mathrm{Nb}$ & $\mathrm{Zr}$ & $\mathrm{Y}$ & $\mathrm{Sr}$ & $\mathrm{Rb}$ & Ga & $\mathrm{Zn}$ & $\mathrm{Ni}$ & Th & Sc & $\mathrm{v}$ & $\mathrm{Cr}$ & Co & $\mathrm{Cu}$ & $\mathrm{Ba}$ & $\mathrm{La}$ & $\mathrm{Ce}$ & $\mathrm{Nd}$ \\
\hline 150 & $808 \mathrm{C}-85 \mathrm{R}-1.59-61$ & 7.8 & 74.2 & 482.6 & 804.0 & 78.4 & 4.7 & 60.8 & 37.6 & 19.0 & 20.0 & 50.9 & 22.9 & 2.5 & 66.7 & 8278.6 & 177.0 & 235.6 & 34.7 \\
\hline 139 & $808 \mathrm{C}-85 \mathrm{R}-2.68-70$ & 17.8 & 164.9 & 24.2 & 97.8 & 166.3 & 21.7 & 103.9 & 63.9 & 16.0 & 11.1 & 132.3 & 76.7 & 22.9 & 60.2 & 629.0 & 21.3 & 64.3 & 18.8 \\
\hline 211 & $808 \mathrm{C}-86 \mathrm{R}-2,110-112$ & 3.9 & 99.1 & 219.0 & 460.9 & 55.5 & 13.0 & 57.9 & 34.8 & 21.4 & 28.2 & 57.5 & 32.4 & 4.6 & 60.6 & 578.4 & 76.5 & 154.5 & 43.7 \\
\hline 51 & $808 C-87 R-1.78-79$ & 17.0 & 149.5 & 31.1 & 97.7 & 142.9 & 25.7 & 107.5 & 49.6 & 6.9 & 10.8 & 124.6 & 69.5 & 21.9 & 72.0 & 489.4 & 31.6 & 61.4 & 25.4 \\
\hline 190 & $808 \mathrm{C}-88 \mathrm{R}-2.21-24$ & 13.5 & 103.3 & 58.2 & 223.9 & 103.5 & 14.6 & 73.0 & 42.8 & 12.2 & 17.7 & 96.2 & 45.8 & 9.3 & 74.0 & 563.6 & 30.5 & 72.9 & 19.9 \\
\hline 197 & $808 \mathrm{C}-89 \mathrm{R}-1.58-60$ & -1.0 & -1.0 & -1.0 & -1.0 & -1.0 & -1.0 & -1.0 & -1.0 & -1.0 & -1.0 & -1.0 & -1.0 & -1.0 & -1.0 & -1.0 & -1.0 & -1.0 & -1.0 \\
\hline 155 & $808 \mathrm{C}-89 \mathrm{R}-1,126-128$ & 12.6 & 139.6 & 21.7 & 233.8 & 147.6 & 19.1 & 93.3 & 31.5 & 14.0 & 13.9 & 110.4 & 64.3 & 12.6 & 66.2 & 456.4 & 19.1 & 55.0 & 18.9 \\
\hline 154 & $808 \mathrm{C}-90 \mathrm{R}-1,98-100$ & 14.7 & 149.4 & 21.8 & 150.3 & 149.0 & 18.7 & 99.1 & 23.3 & 17.1 & 14.8 & 112.6 & 68.5 & 16.3 & 60.7 & 540.0 & 24.7 & 64.9 & 20.3 \\
\hline 141 & $808 C-92 R-1.110-113$ & 9.4 & 141.6 & 31.4 & 146.5 & 152.9 & 19.8 & 103.9 & 50.1 & 15.1 & 11.9 & 111.9 & 71.5 & 19.0 & 66.3 & 553.6 & 25.9 & 70.3 & 16.4 \\
\hline 105 & $808 \mathrm{C}-92 \mathrm{R}-\mathrm{CC}, 8-10$ & 8.8 & 115.1 & 32.6 & 186.3 & 115.7 & 15.7 & 71.1 & 32.3 & 18.8 & 19.6 & 81.0 & 50.1 & 9.5 & 59.3 & 747.7 & 23.6 & 46.4 & 12.4 \\
\hline 182 & $808 C-93 R-1.76-78$ & 12.9 & 168.4 & 28.7 & 103.6 & 153.5 & 21.0 & 103.1 & 31.3 & 22.7 & 15.5 & 123.7 & 70.0 & 15.6 & 90.0 & 495.7 & 27.6 & 69.6 & 27.2 \\
\hline so & $808 \mathrm{C}-94 \mathrm{R}-1.92-94$ & 18.1 & 161.3 & 25.2 & 97.2 & 151.5 & 26.1 & 104.6 & 36.8 & 9.7 & 12.7 & 120.6 & 80.6 & 17.5 & 120.1 & 447.7 & 25.7 & 69.2 & 19.2 \\
\hline 187 & $808 \mathrm{C}-94 \mathrm{R}-1.117-119$ & 17.0 & 165.1 & 27.9 & 106.1 & 167.1 & 23.2 & 105.2 & 35.3 & 12.6 & 11.9 & 120.9 & 79.3 & 16.8 & 59.0 & 481.1 & 24.2 & 75.5 & 16.9 \\
\hline 175 & $808 \mathrm{C}-95 \mathrm{R}-1.22-24$ & 16.3 & 152.8 & 26.2 & 104.8 & 148.5 & 20.4 & 102.5 & 34.7 & 6.6 & 14.6 & 137.6 & 68.4 & 14.3 & 66.3 & 427.5 & 28.2 & 79.3 & 20.4 \\
\hline 79 & $808 C-95 R-1,131-133$ & 10.0 & 109.4 & 59.7 & 252.2 & 116.7 & 15.1 & 81.4 & 32.9 & 25.5 & 20.4 & 71.3 & 44.1 & 10.7 & 37.7 & 645.9 & 48.2 & 58.9 & 20.2 \\
\hline 178 & $808 \mathrm{C}-96 \mathrm{R}-1,40-42$ & 15.0 & 152.3 & 31.1 & 92.7 & 162.6 & 23.9 & 105.2 & 48.4 & 12.5 & 13.7 & 125.1 & 75.7 & 21.4 & 54.3 & 440.4 & 30.7 & 73.5 & 17.3 \\
\hline 52 & $808 \mathrm{C}-97 \mathrm{R}-1.45-46$ & 14.6 & 157.5 & 34.0 & 117.8 & 159.7 & 22.3 & 111.9 & 51.2 & 8.9 & 15.0 & 132.4 & 76.8 & 18.8 & 83.0 & 694.3 & 35.2 & 71.2 & 29.2 \\
\hline 129 & 808 C $-98 R-1-A, 91-92$ & 12.7 & 183.3 & 85.4 & 127.2 & 210.8 & 21.9 & 103.1 & 35.5 & 9.2 & 19.6 & 134.4 & 77.7 & 21.0 & 113.0 & 882.1 & 46.5 & 141.7 & 63.3 \\
\hline 133 & 808C-98R-1-B. $91-92$ siderite? & 14.2 & 181.0 & 34.2 & 98.3 & 230.8 & 21.9 & 98.3 & 27.3 & 9.5 & 15.1 & 124.2 & 64.5 & 18.6 & 58.8 & 604.5 & 31.7 & 66.5 & 18.5 \\
\hline 164 & $808 \mathrm{C}-99 \mathrm{R}-1,86-88$ & 16.8 & 163.8 & 27.7 & 87.1 & 191.3 & 20.4 & 110.8 & 38.1 & 25.8 & 10.2 & 125.5 & 73.5 & 21.6 & 63.0 & 512.0 & 23.3 & 70.8 & 17.8 \\
\hline 198 & $808 \mathrm{C} \cdot 100 \mathrm{R}=1,39-41$ & 11.9 & 152.1 & 31.9 & 104.2 & 158.0 & 18.7 & 79.3 & 43.7 & 10.9 & 13.2 & 95.2 & 70.9 & 17.0 & 41.2 & 561.7 & 22.1 & 61.0 & 20.7 \\
\hline 44 & $808 C-101 R-1,85-87$ & 8.1 & 84.0 & 39.8 & 58.3 & 36.6 & 14.5 & 45.1 & 1.7 & 4.3 & 3.8 & 12.7 & 23.7 & 0.0 & 4.9 & 200.1 & 17.2 & 39.9 & 21.6 \\
\hline 200 & $808 \mathrm{C}-101 \mathrm{R}-3.55-57$ & 11.8 & 122.7 & 39.5 & 72.3 & 11.2 & 15,4 & 34.8 & 0.0 & 7.2 & 4.5 & 16.1 & 5.6 & 0.0 & 4.2 & 270.2 & 12.8 & 39.4 & 9.5 \\
\hline 9 & $808 C-101 R-4,62-64$ & 5.5 & 87.5 & 16.1 & 89.4 & 162.5 & 17.5 & 84.0 & 13.1 & 1.4 & 7.5 & 77.7 & 32.5 & 8.3 & 44.4 & 196.1 & 15.1 & 35.5 & 17.8 \\
\hline 135 & $808 \mathrm{C}-102 \mathrm{R}-2.80-82$ & 5.7 & 78.6 & 29.2 & 218.2 & 172.4 & 13.7 & 62.1 & 20.2 & 0.0 & 10.8 & 69.7 & 27.1 & 8.4 & 39.3 & 200.5 & 20.5 & 41.3 & 12.0 \\
\hline 209 & $808 C-102 R-3,133-134$ & 27.3 & 216.8 & 24.6 & 806.3 & 28.8 & 23.8 & 46.2 & 0.0 & 33.5 & 5.5 & 11.9 & 7.1 & 0.0 & 8.9 & 757.6 & 29.8 & 74.3 & 20.4 \\
\hline 173 & $808 C-103 R-1,121-123$ & 11.9 & 124.0 & 32.7 & 49.9 & 230.0 & 18.9 & 91.0 & 28.5 & 19.9 & 10.6 & 113.0 & 55.7 & 14.4 & 75.6 & 723.4 & 22.2 & 55.9 & 17.9 \\
\hline 111 & $808 C-103 R-3,16-18$ & 17.0 & 198.7 & 50.4 & 73.5 & 162.9 & 18.3 & 95.2 & 26.2 & 11.3 & 16.9 & 81.9 & 38.1 & 10.1 & 91.8 & 659.9 & 23.9 & 71.1 & 19.5 \\
\hline 1 & $808 \mathrm{C}-103 \mathrm{R}-3,40-42$ & 8.9 & 295.4 & 52.7 & 785.3 & 4.0 & 19.9 & 26.6 & 0.0 & 10.0 & 11.1 & 27.0 & 5.4 & 0.0 & 3.4 & 841.1 & 25.1 & 64.7 & 29.9 \\
\hline 109 & $808 C-104 R-1.74-75$ & II.I & 133.6 & 34.4 & 320.0 & 181.9 & 19.2 & 86.4 & 31.4 & 4.2 & 13.8 & 95.2 & 49.4 & 15.0 & 62.4 & 593.1 & 17.3 & 45.2 & 11.9 \\
\hline 15 & $808 \mathrm{C}-106 \mathrm{R}-2,103-108$ & 15.9 & 111.0 & 23.4 & 260.3 & 142.5 & 19.2 & 88.2 & 40.8 & 19.9 & 16.8 & 92.6 & 40.3 & 18.1 & 33.7 & 223.3 & 24.4 & 36.8 & 21.0 \\
\hline 39 & $808 C-106 R-2,103-108$ & 10.4 & 100.1 & 25.7 & 262.1 & 133.5 & 12.8 & 92.2 & 43.2 & 24.8 & 13.5 & 77.0 & 45.0 & 20.8 & 32.3 & 218.9 & 24.0 & 41.2 & 23.8 \\
\hline
\end{tabular}


Table 6. Mean values and 1-sigma standard deviations for the REE's determined on the PV8060 from the reference materials analyzed with the Site 808 samples.

\begin{tabular}{|c|c|c|c|c|c|c|c|c|c|c|c|c|}
\hline \multirow[b]{2}{*}{ Element } & \multicolumn{4}{|c|}{ Reference Sample } & \multicolumn{4}{|c|}{ JB-1a } & \multicolumn{4}{|c|}{ SO-1 SO-2 } \\
\hline & Mean & Std. dev. & \%Std. dev. & Number of runs & Mean & Std. dev. & \%Std. dev. & Number of runs & Mean & Std. dev. & \%Std. dev. & Number of runs \\
\hline $\mathrm{La}$ & 36.39 & 3.21 & 8.83 & 6 & 57.98 & 2.79 & 4.81 & 3 & 50.08 & 1.05 & 2.1 & 2 \\
\hline $\mathrm{Ce}$ & 62.82 & 5.01 & 2.97 & 6 & 114.21 & 5.93 & 5.19 & 3 & 123.48 & 2.43 & 1.97 & 2 \\
\hline $\operatorname{Pr}$ & 7.47 & 0.44 & 5.89 & 6 & 13.92 & 0.65 & 4.69 & 3 & 15.13 & 0.47 & 3.11 & 2 \\
\hline $\mathrm{Nd}$ & 26.42 & 2.4 & 9.06 & 6 & 52.73 & 3.33 & 6.32 & 3 & 63.3 & 0.68 & 1.08 & 2 \\
\hline $\mathrm{Sm}$ & 4.84 & 0.32 & 6.59 & 6 & 8.35 & 0.36 & 4.33 & 3 & 12.74 & 0.25 & 1.93 & 2 \\
\hline $\mathrm{Eu}$ & 1.55 & 0.12 & 7.4 & 6 & 1.78 & 0.1 & 5.39 & 3 & 3.82 & 0.07 & 1.79 & 2 \\
\hline Gd & 5 & 0.5 & 9.99 & 6 & 7.02 & 0.37 & 5.26 & 3 & 12.11 & 0.37 & 3.03 & 2 \\
\hline Dy & 4.33 & 0.32 & 7.48 & 6 & 5.06 & 0.23 & 4.56 & 3 & 9.54 & 0.12 & 1.26 & 2 \\
\hline $\mathrm{Er}$ & 2.47 & 0.19 & 7.6 & 6 & 2.64 & 0.09 & 3.45 & 3 & 4.51 & 0.14 & 3 & 2 \\
\hline $\mathrm{Yb}$ & 1.98 & 0.16 & 8.32 & 6 & 2.14 & 0.11 & 5.24 & 3 & 3.46 & 0.08 & 2.18 & 2 \\
\hline $\mathrm{Lu}$ & 0.3 & 0.02 & 5.94 & 6 & 0.31 & 0.02 & 5.76 & 3 & 0.48 & 0.01 & 2.68 & 2 \\
\hline
\end{tabular}

Note: Analyses were for repeated sample preparations and therefore reflect both sample preparation and instrumental precision combined. The analyses are reported on a fully oxidized, volatile-free basis. 
Table 7. ICP/OE analyses for REE's for selected samples from ODP Site 808. Analyses recalculated to include loss on ignition (LOI).

\begin{tabular}{|c|c|c|c|c|c|c|c|c|c|c|c|c|c|c|c|c|c|c|}
\hline Number & Hole & Core & Base $(\mathrm{cm})$ & Depth (mbsf) & $\mathrm{La}$ & $\mathrm{Ce}$ & Pr & $\mathrm{Nd}$ & Sm & Eu & Gd & Dy & Er & $\mathrm{Yb}$ & Lu & $\mathrm{La} / \mathrm{Yb}$ & Ba ICP & $\mathrm{Nd}$ (raw) \\
\hline 6 & $\mathrm{H}$, mud T8 & 1.1 & 61 & 0.61 & 25.20582 & 53.11288 & 6.756625 & 23.684 & 4.54648 & 1.0812 & 4.78611 & 3.448 & 2.19383 & 1.3117 & 194 & 19.216 & 451.60 & 35.335 \\
\hline 9 & H, mud T6 & 5.2 & 21 & 36.51 & 23.37323 & 50.71854 & 6.218668 & 22.321 & 4.59189 & 1.05986 & 4.51993 & 3.50655 & 1.94804 & 1.51814 & 0.22361 & 15.396 & 374.60 & 31.986 \\
\hline 10 & $\mathrm{H}$, mud T7 & 10.2 & 16 & 79.46 & 23.14714 & 50.03829 & 6.025871 & 22.280 & 4.4407 & 1.03925 & 4.32268 & 3.34995 & 1.74967 & 1.4016 & 0.20357 & 16.515 & 370.62 & 31.842 \\
\hline 1 & $\mathrm{X}$, mud T4 & 2.1 & 27 & 120.87 & 25.02938 & 53.9632 & 6.528808 & 23.914 & 4.7711 & 1.11143 & 4.63423 & 3.74382 & 2.00238 & 1.67189 & 0.24697 & 14.971 & 379.40 & 33.703 \\
\hline 2 & $\mathrm{X}$, mud T8 & 10.1 & 136 & 198.76 & 25.52106 & 54.71858 & 6.621415 & 23.854 & 4.68294 & 1.01223 & 4.420239 & 3.34421 & 1.80442 & 1.44309 & 0.2082 & 17.685 & 406.30 & 34.337 \\
\hline 3 & $\mathrm{X}$, mud T8 & 15.1 & 11 & 244.61 & 25.93293 & 56.5773 & 6.507949 & 25.241 & 4.75895 & 1.06679 & 4.75368 & 3.63729 & 1.90917 & 1.5813 & 0.22643 & 16.400 & 442.70 & 36.663 \\
\hline 4 & $\mathrm{X}$, mud T8 & 20.1 & 64 & 288.54 & 28.09798 & 61.72237 & 6.941755 & 26.399 & 5.13645 & 1.11367 & 4.79826 & 3.66133 & 1.86663 & 1.57881 & 0.22831 & 17.797 & 393.20 & 36.544 \\
\hline 7 & $\mathrm{R}$, mudstone & 1.1 & 100 & 299.5 & 19.56879 & 44.65028 & 5.708311 & 20.304 & 4.31489 & 1.00788 & 4.55162 & 2.51356 & 2.11852 & 1.49654 & 0.23113 & 13.076 & 380.30 & 30.116 \\
\hline 5 & $\mathrm{X}, \operatorname{mud} \mathrm{T} 8$ & 25.1 & 29 & 336.29 & 21.02888 & 46.68863 & 5.795912 & 19.083 & 4.17163 & 0.88125 & 4.10568 & 2.93797 & 1.68534 & 1.26001 & 0.19502 & 16.689 & 351.30 & 28.146 \\
\hline 8 & $\mathrm{R}$, mudstone & 5.1 & 38 & 337.28 & 26.02474 & 57.3836 & 6.556127 & 25.230 & 4.86245 & 1.09272 & 4.71695 & 3.56105 & 1.90215 & 1.49896 & 0.21358 & 17.362 & 390.70 & 35.310 \\
\hline II & $\mathrm{R}$, mudstone & 10.1 & 73 & 385.93 & 23.27912 & 51.60208 & 6.359632 & 23.756 & 4.74786 & 1.09805 & 4.899011 & 3.79739 & 2.23283 & 1.66262 & 0.24536 & 14.001 & 378.80 & 33.529 \\
\hline 12 & $\mathrm{R}$, mudstone & 15.1 & 50 & 434.2 & 24.15981 & 53.82382 & 6.443034 & 23.675 & 4.63952 & 1.04945 & 4.5121 & 3.5983 & 1.89833 & 1.58991 & 0.23144 & 15.196 & 387.00 & 33.660 \\
\hline 13 & $\mathrm{R}$, mottle mud & 20.4 & 122 & 487.42 & 22.15208 & 49.69103 & 5.98105 & 22.738 & 4.65613 & 1.09507 & 4.27981 & 3.70166 & 2.04061 & 1.63047 & 0.24147 & 13.586 & 397.40 & 32.991 \\
\hline 14 & R. mudstone & 25.1 & 52 & 530.52 & 24.94615 & 54.75072 & 6.36764 & 23.588 & 4.38125 & 0.99573 & 4.32537 & 3.21846 & 1.64809 & 1.33397 & 0.19172 & 18.701 & 415.30 & 34.303 \\
\hline 15 & $\mathrm{R}$, mudstone & 30.1 & 116 & 579.46 & 25.43581 & 55.03364 & 6.37449 & 23.962 & 4.67668 & 1.04357 & 4.23798 & 3.33389 & 1.64347 & 1.33794 & 0.19594 & 19.011 & 401.20 & 34.313 \\
\hline 16 & R, crumbled? & 33.2 & 20 & 608.6 & 27.14651 & 59.40911 & 6.865552 & 25.472 & 4.98096 & 1.08199 & 3.9691 & 3.51765 & 1.89833 & 1.33816 & 0.19624 & 20.286 & 408.80 & 36.019 \\
\hline 17 & R, mottle mud & 35.5 & 86 & 633.05 & 24.12469 & 50.37546 & 5.960838 & 21.968 & 4.34159 & 1.0077 & 4.27701 & 3.55342 & 1.88893 & 1.68731 & 0.2556 & 14.298 & 371.50 & 31.553 \\
\hline 18 & R. mottle mud & 40.2 & II & 675.81 & 29.31103 & 62.03017 & 7.27521 & 26.264 & 4.91982 & 1.11517 & 4.65023 & 3.43985 & 1.72127 & 1.38356 & 0.20427 & 21.185 & 428.50 & 37.319 \\
\hline 19 & $\mathrm{R}$, mottle mud & 43.2 & 69 & 705.39 & 27.89676 & 61.22523 & 6.921568 & 25.709 & 4.90196 & 1.06156 & 4.62238 & 3.43591 & 1.65647 & 1.3412 & 0.19439 & 20.800 & 432.90 & 36.878 \\
\hline 20 & R, pyrocl sst & 45.3 & 60 & 725.6 & 20.18387 & 43.08026 & 5.268607 & 24.146 & 2.8718 & 1.12499 & 5.08716 & 4.64934 & 2.40775 & 2.75584 & 0.43876 & 7.3240 & 1771.0 & 69.837 \\
\hline 21 & R, mottle mud & 50.3 & 54 & 773.94 & 26.52588 & 55.63246 & 6.424997 & 25.146 & 4.66848 & 1.09924 & 4.72488 & 3.91005 & 2.01024 & 1.8096 & 0.26434 & 14.658 & 382.70 & 35.019 \\
\hline 22 & R. mottle mud & 52.1 & 62 & 790.42 & 28.85009 & 62.39654 & 7.183707 & 27.153 & 4.59138 & 0.98892 & 4.36599 & 3.31777 & 1.57423 & 1.29312 & 0.1886 & 22.310 & 521.50 & 40.608 \\
\hline 23 & $\mathrm{R}$, mottle mud & 55.1 & 101 & 819.71 & 29.53042 & 61.18817 & 6.961075 & 26.350 & 4.66703 & 1.08922 & 4.36169 & 3.17378 & 1.66958 & 1.31172 & 0.18588 & 22.513 & 438.20 & 37.655 \\
\hline 24 & $\mathrm{R}$, mottle mud & 57.2 & 110 & 840.7 & 32.48187 & 67.46812 & 7.816065 & 29.292 & 5.09239 & 1.13781 & 4.83829 & 3.59363 & 1.88336 & 1.52597 & 0.22029 & 21.286 & 452.90 & 40.977 \\
\hline 25 & $\mathrm{R}$, mottle mud & 60.1 & 14 & 866.94 & 28.70824 & 59.93343 & 6.835717 & 25.787 & $\begin{array}{l}4.65399 \\
\end{array}$ & 1.02228 & 4.31851 & 3.29014 & 1.72221 & 1.41271 & 0.20173 & 20.321 & 351.40 & 34.853 \\
\hline 26 & $\mathrm{R}$, mottle mud & 65.1 & 113 & 916.23 & 35.18215 & 72.23592 & 8.256433 & 30.509 & 5.28074 & 1.17895 & 4.89881 & 3.63756 & 1.85902 & 1.55463 & 0.22649 & 22.631 & 494.30 & 43.261 \\
\hline 27 & $\mathrm{R}$, mottle mud & 66.2 & 117 & 927.47 & 35.83366 & 74.26247 & $\begin{array}{l}8.35845 \\
8.250\end{array}$ & 31.350 & 5.42213 & 1.1788 & $\begin{array}{l}4.070621 \\
4.7962\end{array}$ & 3.51399 & 1.80471 & $\begin{array}{l}1.46756 \\
\text {. }\end{array}$ & 0.21482 & 24.417 & 468.30 & 43.432 \\
\hline 28 & $\mathrm{R}$, mottle mud & 67.1 & 147 & 935.97 & 34.82556 & 71.59315 & 8.414363 & 31.069 & 5.55353 & 1.24617 & 5.1316 & 3.94459 & 2.06829 & 1.64666 & 0.24333 & 21.149 & 502.00 & 44.021 \\
\hline 29 & $R$, mottle mud & 68.1 & 48 & 944.68 & 34.54346 & 71.23864 & 8.254707 & 31.554 & 5.38984 & 1.2204 & 4.81869 & 3.72544 & 1.92733 & 1.54692 & 0.22438 & 22.330 & 471.40 & 43.716 \\
\hline 30 & $\mathrm{R}$, decollement & 69.1 & 6 & 953.86 & 33.88381 & 72.76306 & 8.465125 & 32.191 & 6.19577 & 1.56603 & 7.023099 & 5.6922 & 3.29406 & 2.3982 & 0.35341 & 14.129 & 458.70 & 44.026 \\
\hline 31 & $\mathrm{R}$, decollement & 69.3 & 112 & 957.92 & 36.33672 & 73.35169 & 8.497578 & 32.176 & 5.72894 & 1.3349 & 5.88767 & 4.67509 & 2.62292 & 2.18883 & 0.3217 & 16.601 & 522.70 & 45.662 \\
\hline 32 & $\mathrm{R}$, decollement & 69.4 & 68 & 958.98 & 32.69526 & 65.44059 & 7.790429 & 28.561 & 5.02995 & 1.09076 & 4.57677 & 3.18591 & 1.58774 & 1.09916 & 0.15576 & 29.746 & 437.40 & 39.846 \\
\hline 33 & $\mathrm{R}$, decollement & 69.5 & 63 & 960.42 & 38.54763 & 77.19643 & 9.025819 & 34.648 & 6.46384 & 1.51924 & 7.12265 & 6.71667 & 4.34408 & 4.21062 & 0.64281 & 9.1549 & 526.70 & 48.237 \\
\hline 34 & $\mathrm{R}$, mottle mud & 70.1 & 43 & 963.83 & 34.18828 & 69.34116 & 8.11881 & 30.727 & $\begin{array}{l}0.4031143 \\
5.2113\end{array}$ & $\begin{array}{l}1.17346 \\
\end{array}$ & 5.10571 & 3.83333 & 1.86647 & 1.50903 & 0.21836 & 22.656 & 604.30 & 46.318 \\
\hline 35 & $\mathrm{R}$, mottle mud & 71.1 & 33 & 973.43 & 34.6924 & 72.98605 & 8.301262 & 32.420 & 6.00345 & 1.37978 & 6.07771 & 5.0026 & 2.71138 & 2.23476 & 0.33005 & 15.524 & 478.40 & 44.763 \\
\hline 36 & $\mathrm{R}$, mottle mud & 71.3 & 92 & 977.02 & 36.76616 & 74.1359 & 8.298916 & 31.927 & 5.31167 & $1.1591^{\circ}$ & 4.74375 & 3.31919 & 1.50511 & 1.2024 & 0.16964 & 30.577 & 575.70 & 46.780 \\
\hline 37 & $\mathrm{R}$, mottle mud & 75.1 & 117 & 1012.52 & 33.14368 & 70.661 & 7.804063 & 29.562 & 5.0798 & 1.08422 & 4.50358 & 3.3516 & 1.59756 & 1.35044 & 0.19635 & 24.543 & 508.80 & 42.689 \\
\hline 38 & $\mathrm{R}$, mottle mud & 78.4 & 95 & 1044.52 & 34.81438 & 72.52616 & 7.947577 & 30.571 & 5.04251 & 1.07416 & 4.64257 & 3.36155 & 1.61471 & 1.39363 & 0.2 & 24.981 & 566.60 & 45.190 \\
\hline 39 & R, carbonate & 80.2 & 78 & 1060.11 & 186.6305 & 298.4738 & 30.59836 & 117.38 & 20.87868 & 6.4967 & 33.68956 & 31.86821 & 21.18133 & 14.61138 & 2.16009 & 12.773 & 1004.4 & 143.29 \\
\hline 40 & $\mathrm{R}$, mottle mud & 82.1 & 107 & 1077.61 & 31.84775 & 67.31701 & 7.574239 & 28.026 & 4.75189 & 1.05185 & 4.29493 & 2.99711 & 1.43972 & 1.14372 & 0.16949 & 27.846 & 438.90 & 39.350 \\
\hline 41 & $\mathrm{R}$, mottle mud & 85.2 & 28 & 1100.12 & 30.03079 & 60.44579 & 6.7477 & 25.187 & 4.21445 & 0.93294 & 4.03464 & 3.29767 & 1.79469 & 1.7705 & 0.27329 & $\begin{array}{l}16.9620 \\
16.962\end{array}$ & 538.30 & 39.075 \\
\hline 42 & $\mathrm{R}$, mottle mud & 88.1 & 41 & 1127.3 & 34.75351 & 72.83688 & 8.238038 & 30.8 & 5.38884 & 1.19998 & 5.12069 & 3.88196 & 2.05594 & 173055 & 0.25766 & 20. & 469.70 & 43.004 \\
\hline 43 & $\mathrm{R}$, mottle mud & 90 & $\begin{array}{l}41 \\
59\end{array}$ & 1 & 33.93 & 70.15 & 8.097 & 29. & 5.43 & 1.17 & 5.26 & 4.16 & 2.36493 & & & 16.724 & 425.40 & 40.460 \\
\hline 44 & $\mathrm{R}$, mottle mud & 92.1 & 140 & 1167.01 & 34.70989 & 74.84795 & 8.369534 & 30.885 & 5.61517 & 1.22766 & 5.12884 & 4.07034 & 1.69649 & 1.9057 & 0.25016 & 18.214 & 461.70 & 42.797 \\
\hline 45 & $\mathrm{R}$, mottle mud & 94.2 & 46 & 1186.81 & 34.94095 & 72.88142 & 8.292532 & 29.296 & 5.11196 & 1.0935 & 4.89825 & 3.21971 & 1.74645 & 1.03646 & 0.15138 & $\begin{array}{l}33.712 \\
\end{array}$ & 472.70 & 41.492 \\
\hline 46 & $\mathrm{R}$, mottle mud & 97.1 & 25 & . & 22.2864 & 510 & 5.853738 & 23.2 & 4.12 & 0.97504 & 4.04551 & 3.27 & 1.68 & 1.49398 & 0.22286 & 14.918 & 483.30 & 35.764 \\
\hline 48 & hy mud & 100.1 & 73 & & & 30. & 3.7 & & 2.6 & & 11.7 & & 2.9 & & & 8.5454 & 21 & 17.541 \\
\hline 49 & $R$, ash & 101.2 & 21 & 1253.91 & 51.15969 & 128.3653 & 15.34446 & 65.531 & 12.43248 & 3.78772 & 20.30998 & 10.00324 & 4.9236 & 2.81103 & 0.48428 & 18.200 & 946.20 & 89.943 \\
\hline 47 & $R$, green mud & 102.1 & 30 & 1261.81 & 30.50893 & 61.25724 & 7.255175 & 27.291 & 4.82266 & 0.95909 & 5.080659 & 4.81216 & 2.94636 & 2.42155 & 0.34715 & 12.599 & 374.30 & 36.948 \\
\hline
\end{tabular}



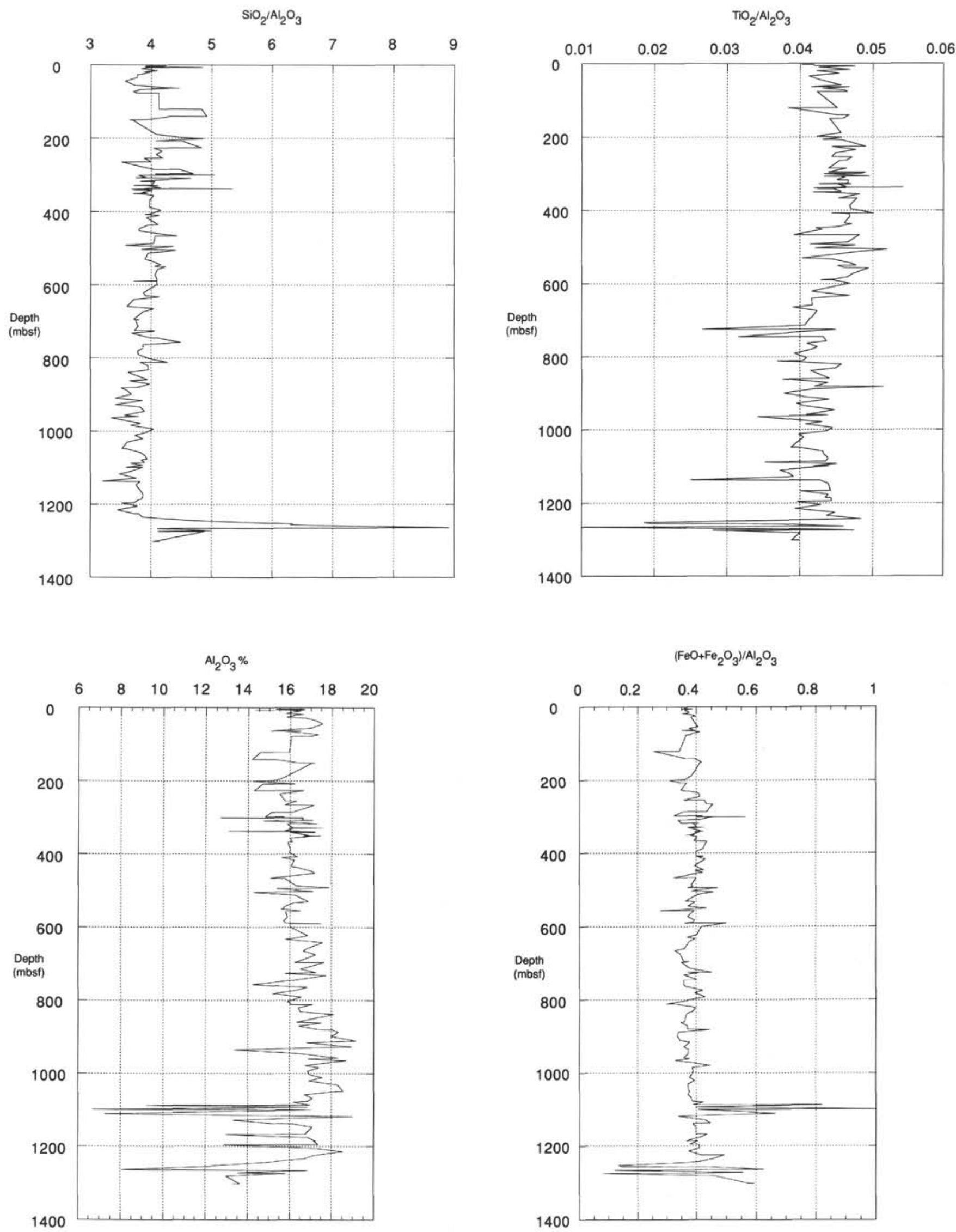

Figure 1. Element and element oxide abundances vs. depth (mbsf) for Site 808. Graphs show element and element oxide abundances normalized to $\mathrm{Al}_{2} \mathrm{O}_{3}$ vs. depth. 

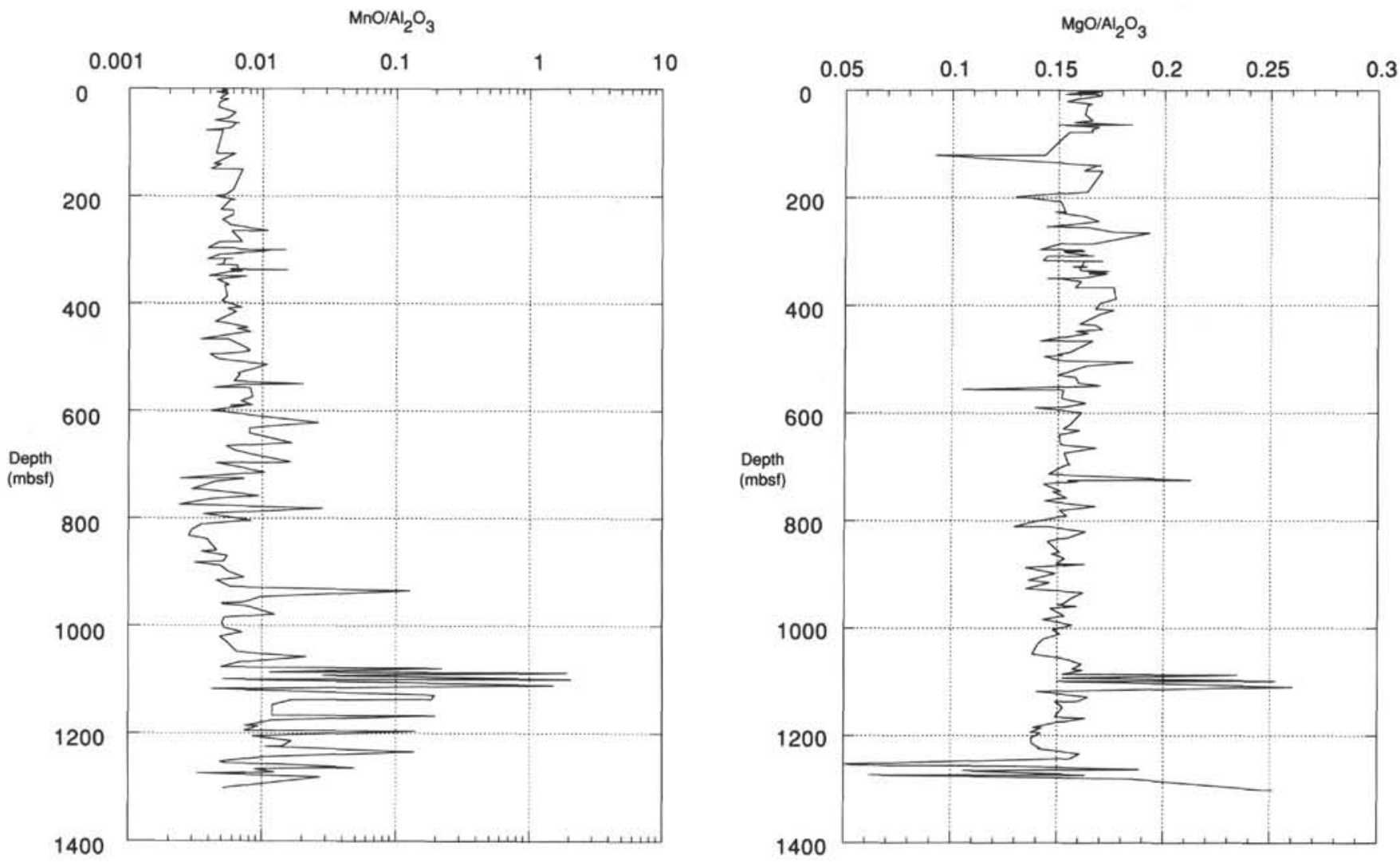

$\mathrm{CaO} / \mathrm{Al}_{2} \mathrm{O}_{3}$

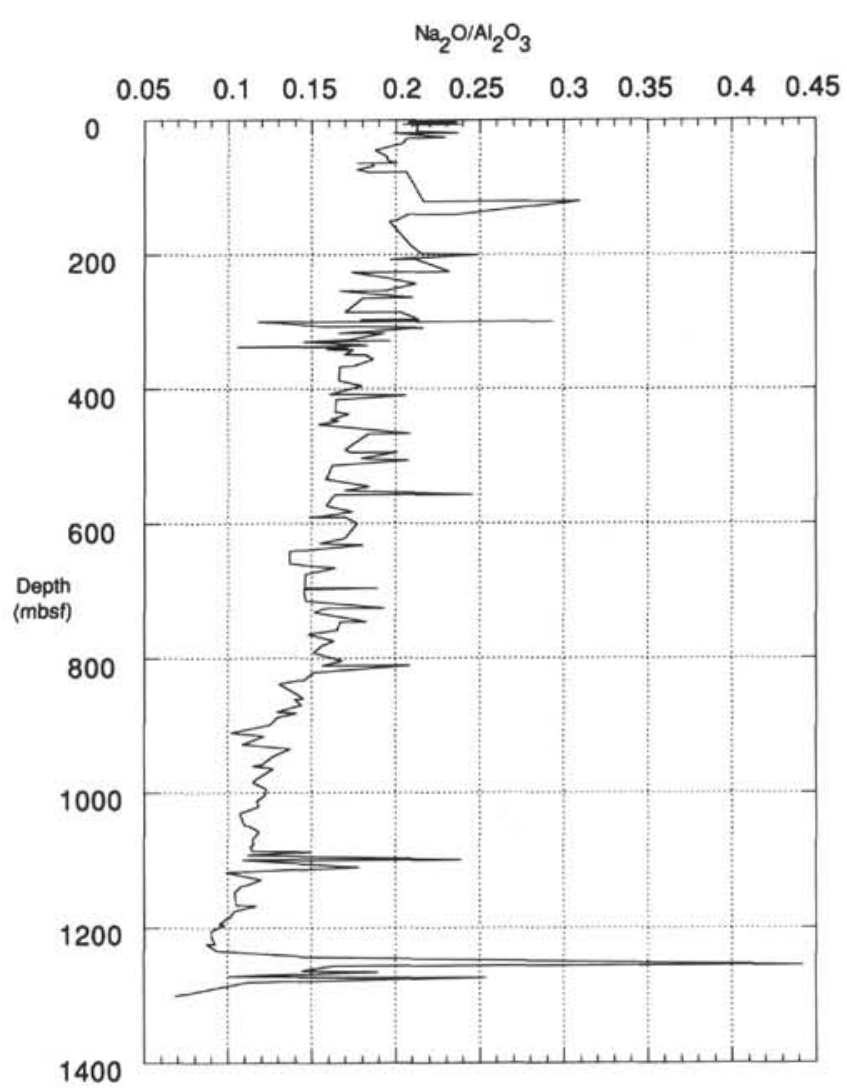

Figure 1 (continued). 
$\mathrm{K}_{2} \mathrm{O} / \mathrm{Al}_{2} \mathrm{O}_{3}$

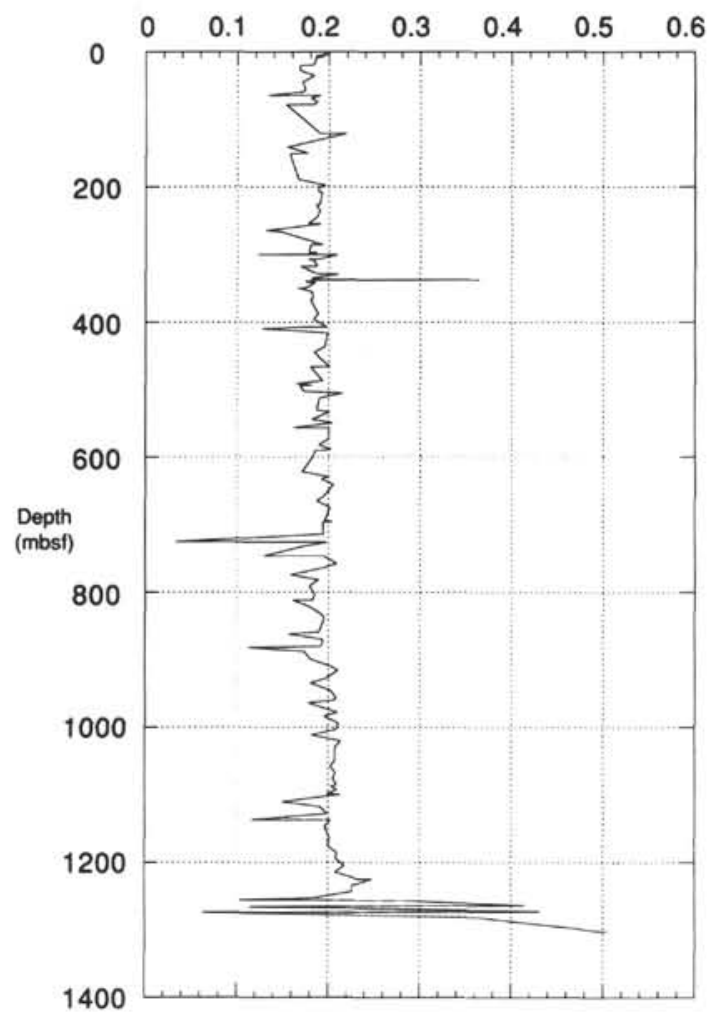

Loss on Ignition \%

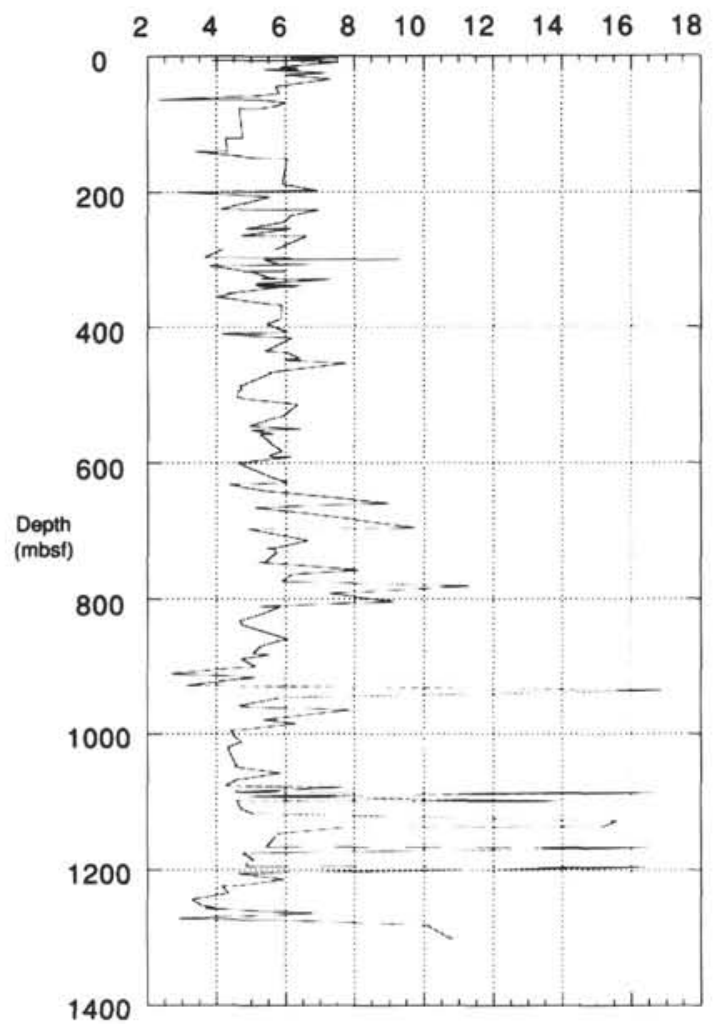

$\mathrm{P}_{2} \mathrm{O}_{5} / \mathrm{Al}_{2} \mathrm{O}_{3}$
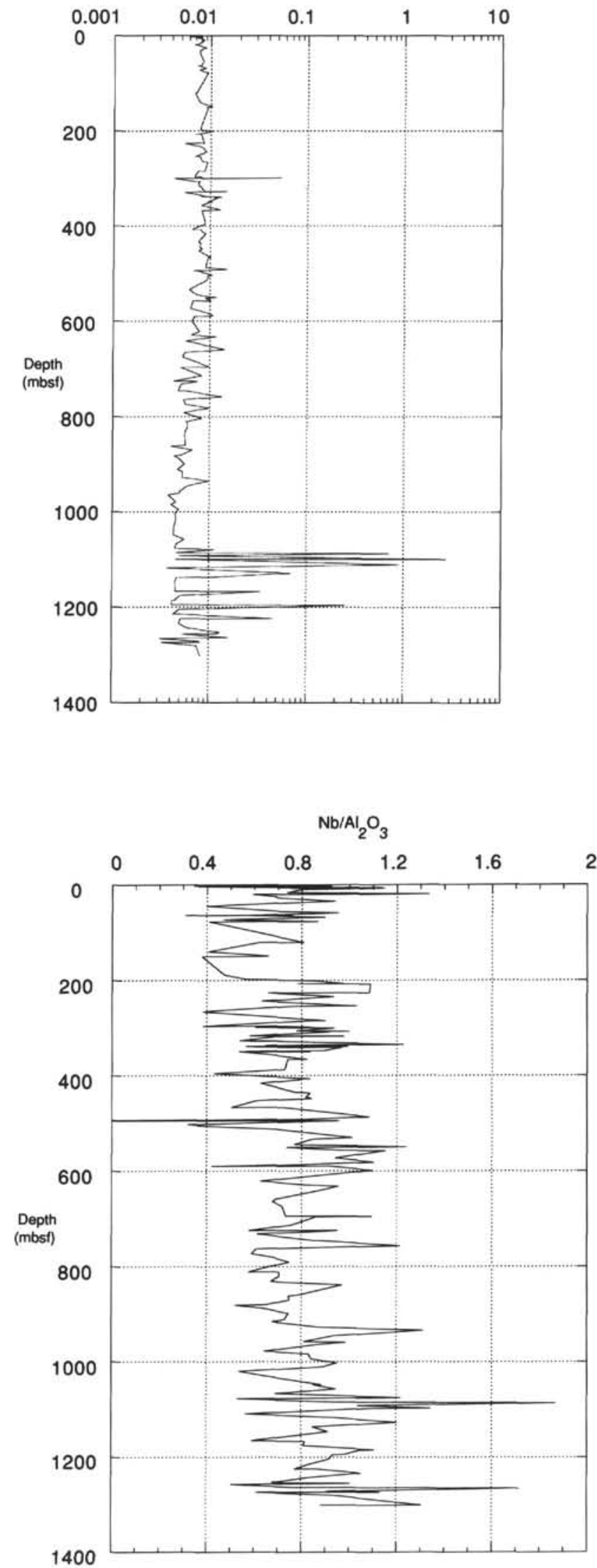

Figure 1 (continued). 

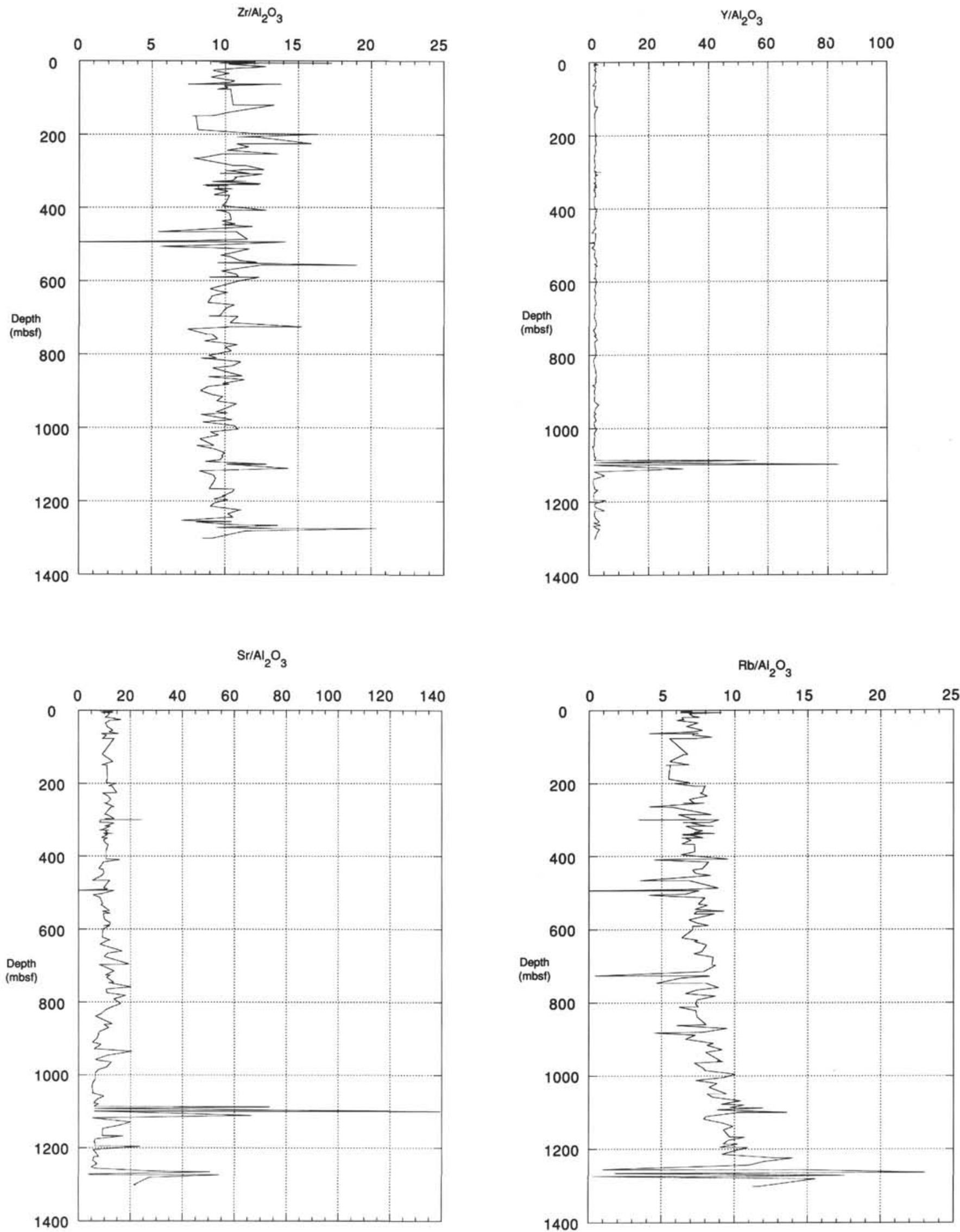

Figure 1 (continued). 

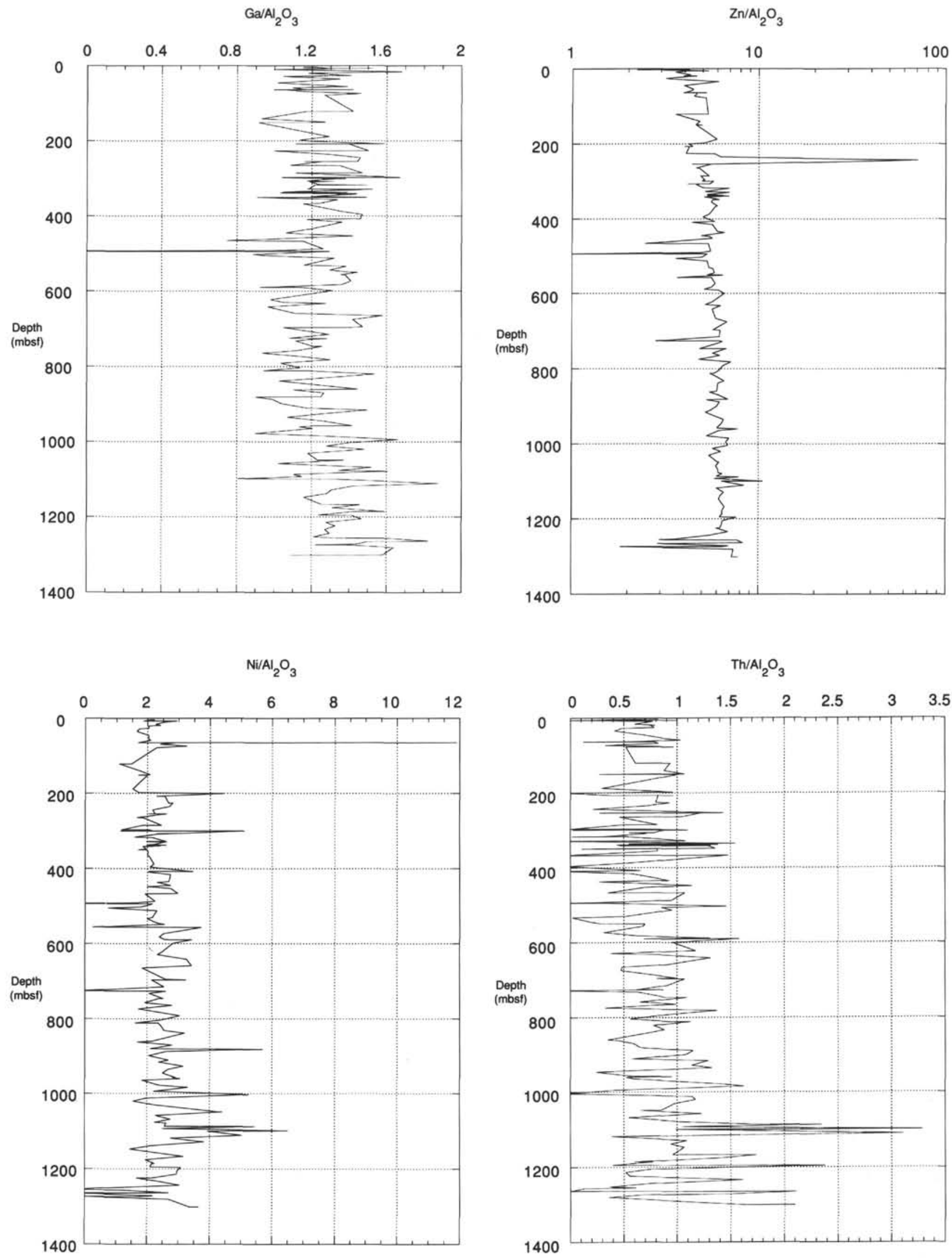

Figure 1 (continued). 

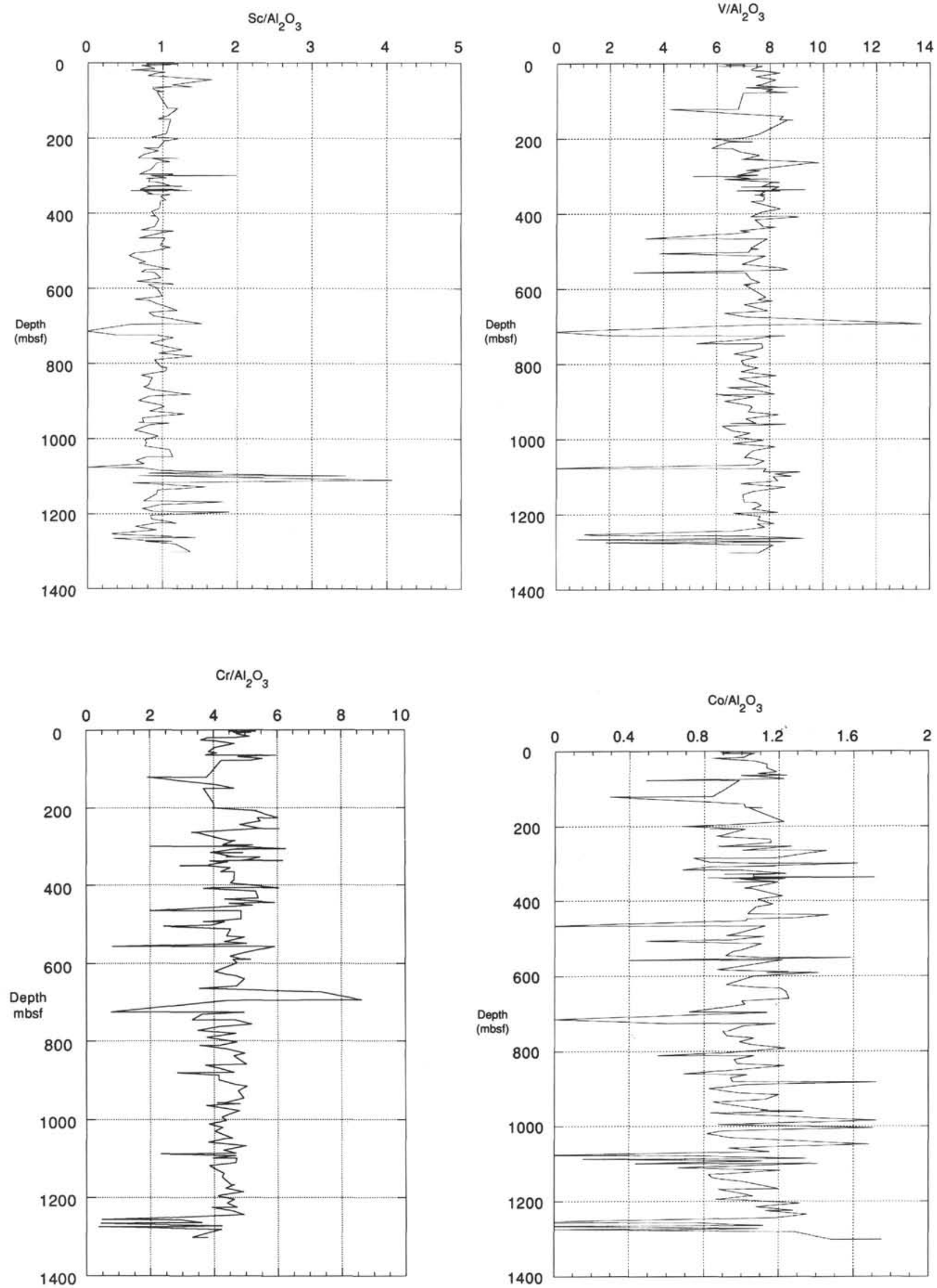

Figure 1 (continued). 

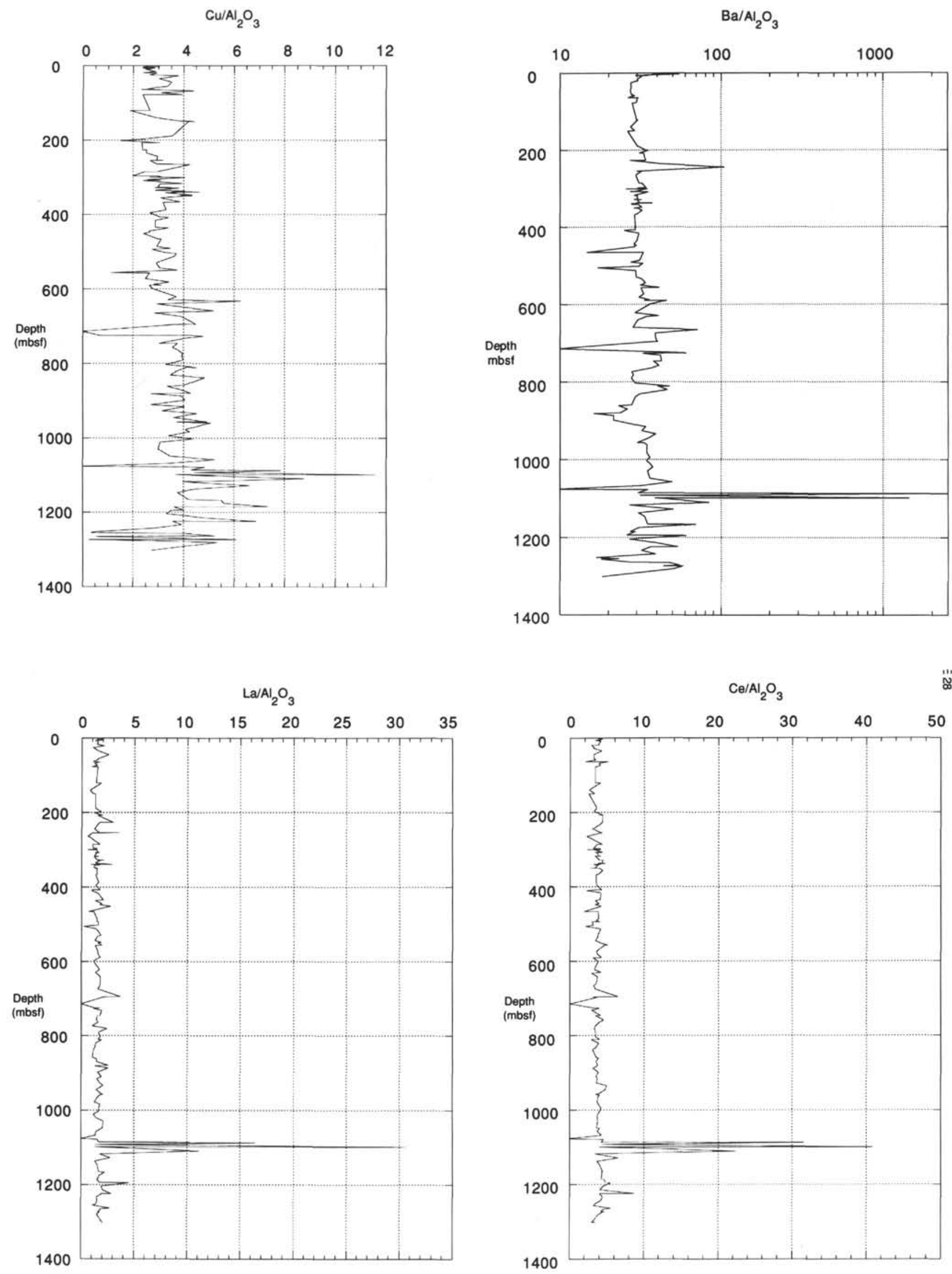

Figure 1 (continued). 


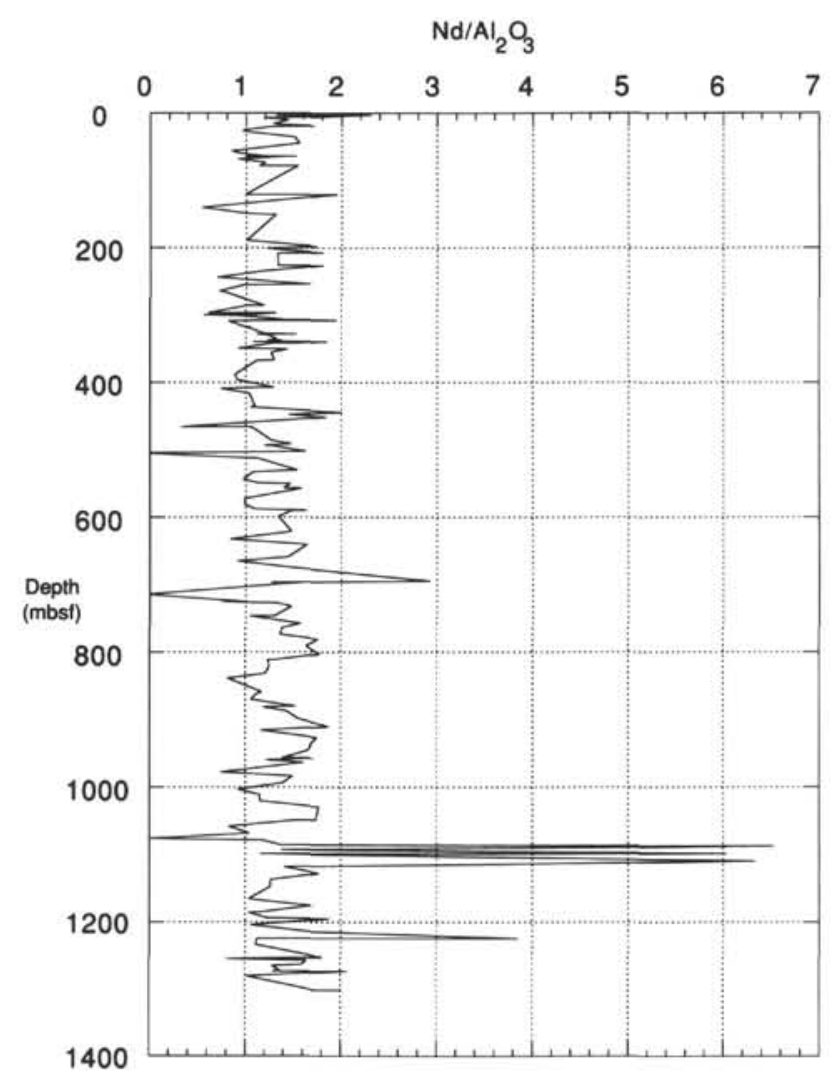

Figure 1 (continued). 

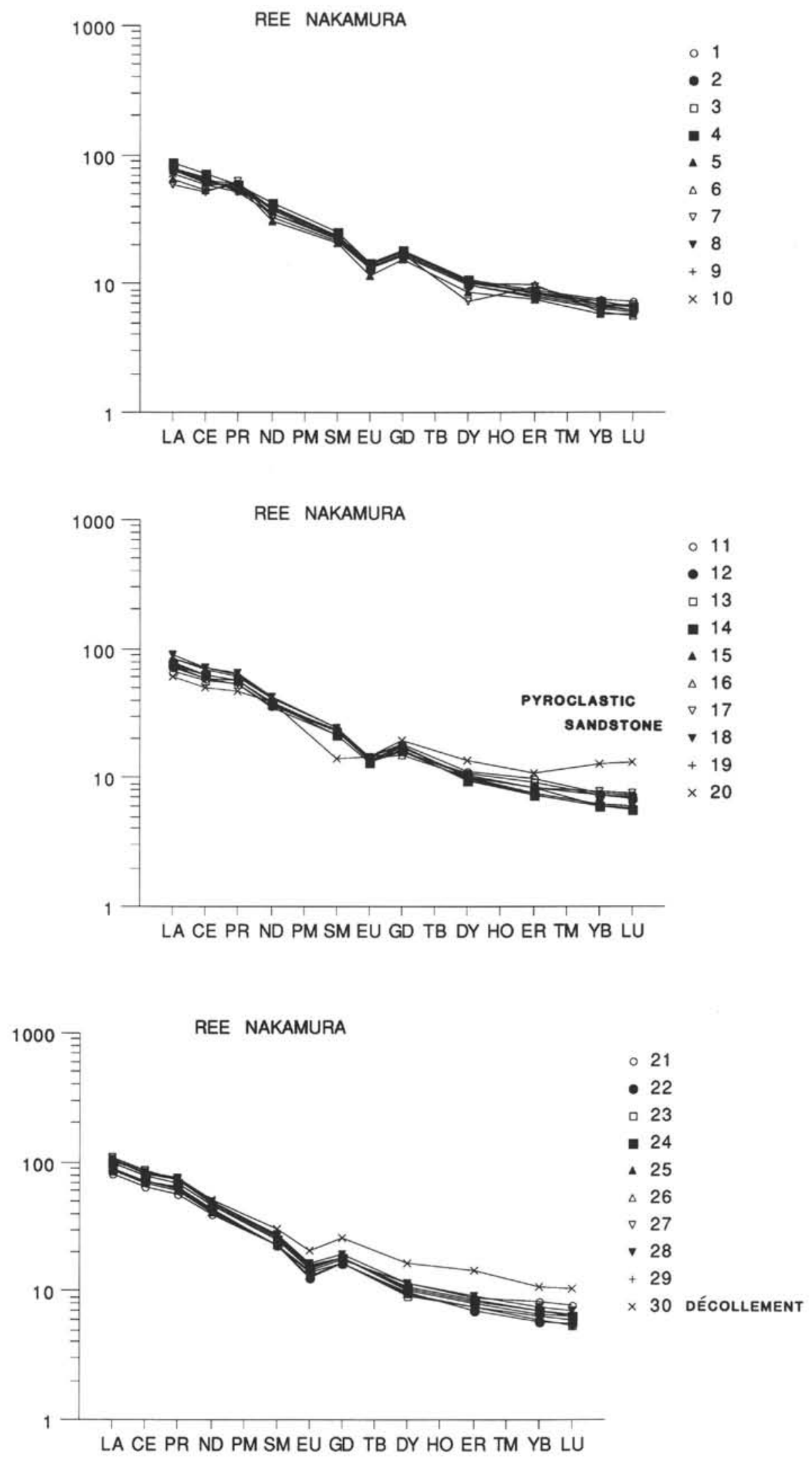

Figure 2. Chondrite normalized (Nakamura, 1974) REE abundances for 49 sediment samples, mainly mud, throughout Site 808 , grouped for clarity in tens. Note that the few primary volcanic sands and ashes analyzed show typical higher total abundances of REE, with pyroclastic sand showing large negative Eu anomaly. Also note that three out of four mud samples from the $\sim 20$-m-thick décollement show enhanced heavy REE abundances. 

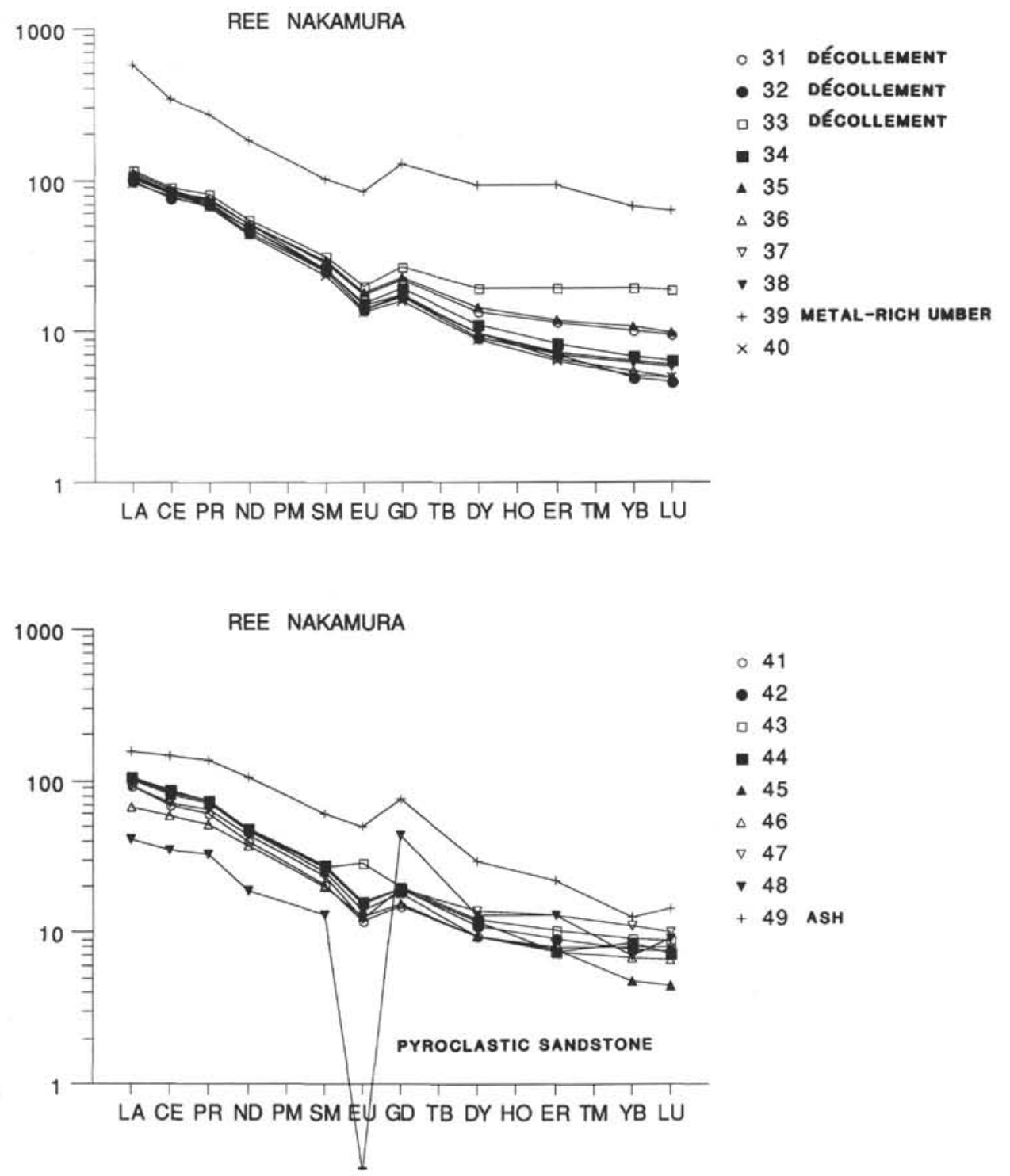

Figure 2 (continued). 


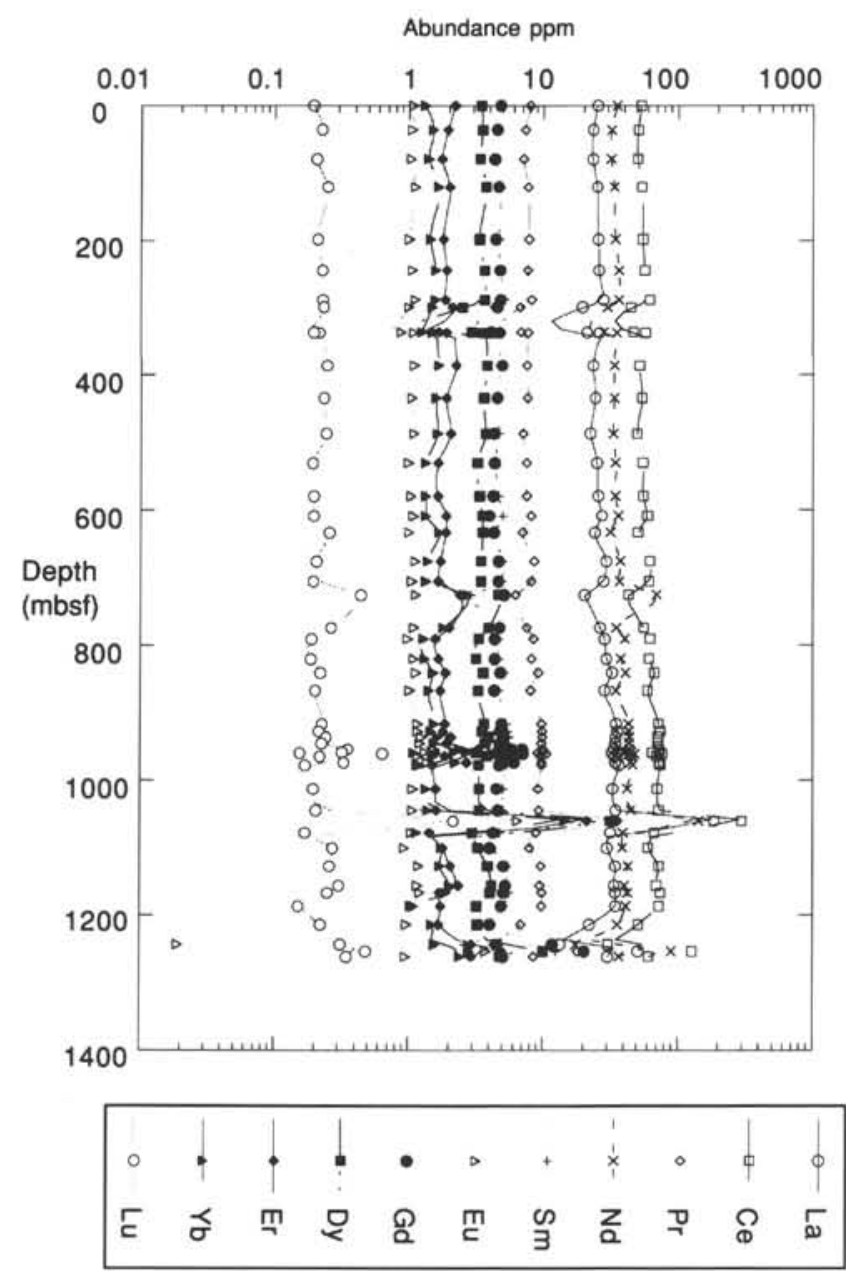

Figure 3. Rare-earth-element abundances (ppm) vs. depth (mbsf) for Site 808. Logarithmic axes used to clarify abundance data. Note the dramatic increase in abundances in the interval at $\sim 1060 \mathrm{mbsf}$, associated with a metalliferous brown mud, also enriched in $\mathrm{Ca}, \mathrm{Fe}, \mathrm{Mg}, \mathrm{Mn}$, and $\mathrm{P}$ oxides. 